\title{
Multiscale process simulation of residual stress fields of laser beam welded precipitation hardened AA6082
}

\author{
Jan Herrnring ${ }^{\mathrm{a}}$, Peter Staron ${ }^{\mathrm{a}}$, Nikolai Kashaev ${ }^{\mathrm{a}}$, Benjamin Klusemann ${ }^{\mathrm{a}, \mathrm{b}}$ \\ ${ }^{a}$ Helmholtz-Zentrum Geesthacht, Institute of Materials Research, Max-Planck-Straße 1, 21502 Geesthacht, Germany \\ ${ }^{b}$ Leuphana University of Lüneburg, Institute of Product and Process Innovation, Volgershall 1, 21339 Lüneburg, Germany
}

\begin{abstract}
In this study, a multiscale modelling approach for the determination of residual stresses for the laser beam welded, precipitation hardened aluminium alloy AA6082-T6 is presented and applied. The material behaviour is described by an elasto-visco-plastic material model, specially suited for fusion welding processes. The microstructure evolution during the welding process has a direct influence on the macroscopic mechanical properties. The modelling approach accounts for the change in the microstructure via a Kampmann-Wagner Numerical model which takes into account the kinetics of the precipitates. The macroscopic mechanical properties are determined via classic dislocation theory, which accounts for the interaction between dislocations and precipitates. The temperature field of the welding process is described by a highly efficient semi-analytical approach. The solution of the temperature field in connection with a three dimensional moving heat source is achieved by using the method of Green's functions. By employing the method of Green's functions, it is possible to reduce the numerical effort significantly. The results of this modelling approach are compared to temperature, hardness as well as residual stress measurements, obtained from synchrotron X-ray diffraction, for welded sheets to clarify the accuracy of the applied model.
\end{abstract}

Key words: Welding, Green's function, Residual stresses, Kampmann-Wagner Numerical model, Multiscale approach

\section{Introduction}

Simulation methods for welding processes are of high importance for the manufacturing industry to understand the complex multi-physics phenomena that are involved. Welding processes are characterised by physical problems on different length scales. Obviously, the change between the solid and liquid phase is crucial, but besides this, the effect of phase transformation in the solid state needs to be accounted for. Especially for welding of precipitation hardenable aluminium alloys, the solid state precipitation process is of great significance because the mechanical properties depend strongly on the underlying microstructure (Esmaeili et al., 2003). On the basis of classic continuum theory, the simulation of residual stress fields that evolve during the welding process has gained large attention in the area of process simulation in the last decades (Lindgren, 2001). Nevertheless, simulations without taking into account the change in the microstructure cannot predict residual stress fields in the fusion and the heat affected zone (HAZ) with sufficient accuracy. Precipitation hardened aluminium alloys like, e.g., aluminium-magnesium-silicon alloys, are intensively used in automotive and aircraft industry. The simulation of welding-induced residual stress fields in these alloys has been investigated by Moraitis and Labeas (2008) and Nélias et al. (2010) via modelling approaches based on classic continuum mechanics. Microstructure as well as mechanical properties of laser beam welded AA6056 has been investigated by Pakdil et al. (2011) from experimental perspective. Small-angle neutron scattering as well as synchrotron X-ray diffraction has been used by Staron et al. $(2006,2009)$ for investigation of the precipitates and

Email address: jan.herrnringehzg.de (Jan Herrnring)

Preprint submitted to Materialia 
residual stress fields of laser beam welded AA6056. These investigations reveal dissolution of precipitates as well as residual stress fields showing a 'M' profile.

As a starting point for the calculation of residual stress fields, the transient temperature field of the welding process is required. The simulation of the temperature field for fusion welding processes in the solid and liquid phases, where multi-physical phenomena occurring during welding, is a difficult task and a long standing issue. A multi-physics simulation, which accounts for the fluid dynamics of the molten material was recently reported by Yang et al. (2017). As outlined in Dal and Fabbro (2016), the appropriate simulation method depends decisively on the objective of the study. For calculation of residual stress fields, the temperature field below the melting temperature is of major concern. For this objective, one of the most frequently applied methods is the usage of the double ellipsoidal heat source proposed by Goldak et al. (1984), which is introduced in the nonlinear heat conduction equation.

Heat source models commonly introduce unknown parameters that must be identified by comparison to experimental data. Time-consuming and computational-intensive numerical methods such as the finite element method require a significant amount of time and computational power. In particular, the variation of parameters in an inverse optimization procedure requires a large number of simulations. To reduce the computational costs drastically, a semi-analytical approach is used based on the method of Green's functions. This solution method is highly analytic and thus reduces the numerical effort significantly. One of the early contributions towards the usage of Green's functions for heat conduction in combination with laser processes have been given already by Cline and Anthony (1977). In our contribution, the method of Green's function is used to solve an initial boundary value problem of the heat equation. A quite similar methodology has been used by Karkhin et al. (2011) for simulating welding processes via the source method. Their approach is used as a starting point and extended to different boundary conditions through the usage of Green's functions.

The residual stress field is obtained by solving of a mechanical boundary value problem using the finite element method. A material model that accounts for the special characteristics occurring during welding is employed via a multiscale approach. The phase change is modelled by a material model originally introduced for welding and shapedmetal deposition processes by Chiumenti et al. (2010). This material model is capable of changing its constitutive material behaviour from viscoplastic below the solidus temperature to viscous above the liquidus temperature.

In addition to the phase change between solid and liquid, the solid-state precipitation kinetics has to be considered. Numerous precipitates in the fusion zone and HAZ become unstable and dissolve in the aluminium matrix during the process. The decrease in particle volume density leads to a significant loss of strength. To account for the precipitation kinetics, a Kampmann-Wagner Numerical (KWN) model based on the extensions by Myhr and Grong (2000) and Myhr et al. $(2001,2004)$ is used. This model accounts for nucleation, growth and dissolution of precipitates. The KWN simulation approach has become an important microstructure simulation method especially for precipitation hardening aluminium alloys. Especially Mg-Si-Al-alloys have been investigated intensively (Myhr et al., 2004; Bardel et al., 2014; Du et al., 2017). The KWN simulation approach facilitates the simulation of complex non-isothermal phase transformations and is very efficient compared to other simulation approaches such as the phase field method. Nonetheless, practical implementations are rarely found in literature. Valuable information about implementation is given by Myhr and Grong (2000) and Perez et al. (2008). The numerical efficiency and the simple handling of nucleation, which characterizes the great advantage of the KWN model compared to other microstructure models, makes the KWN model to a natural coupling partner for finite element simulations. Recently, such a coupling procedure has been used by Bardel et al. (2016a) for investigation of welds made from AA6061-T6.

In the following, the general research approach for the numerical and the experimental part is presented. Afterwards, the incorporated submodels are presented in detail and the simulation results are compared to experimental data obtained from X-ray diffraction measurements of laser beam welded specimens.

\section{Experimental and numerical approach}

\subsection{Multiscale modelling approach}

A characteristic of temperature fields during laser beam welding is the high degree of inhomogeneity. These large thermal gradients lead to significant stresses because hot material is hindered from expansion or consolidation by the cold surrounding material. Besides the occurrence of thermal stresses, the temperature rise leads to changes 


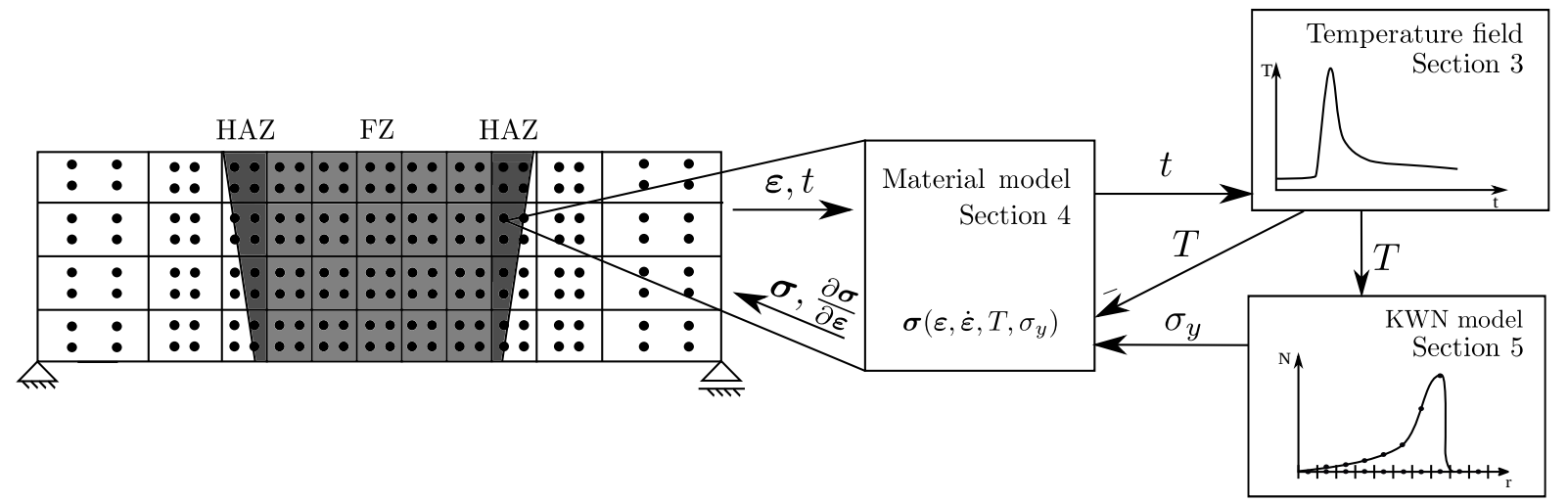

Figure 1: Multiscale simulation strategy for calculation of residual stresses by finite element method. Elements are sketched in combination with the associated integrations points for the macroscopic structure. At each integration point, the material model is called, representing the microscale of this problem. The material model obtains the temperature from the temperature field model as well as the current yield strength by means of the KWN model; both models are called from the material model.

in the microstructure in the fusion zone and the HAZ. The growth and dissolution of precipitates is described by a microscopic KWN model. The KWN model is incorporated in a phenomenological macroscopic material model that describes the phase change between liquid and solid state. This phenomenological material model is capable of changing the behaviour from elasto-viscoplastic for the solid case to purely viscous in the liquid case. The coupling of the different submodels is summarized in Fig.1.

An implicit finite element calculation schema is used in our approach to obtain the values of the next time step. For this purpose, the information of the local deformation are provided to the material routine at each integration point of the macrostructure. The material routine calculates the stresses $\sigma$, based on the coupled plasticity-KWN model in combination with the temperature field model. Finally, the material model determines the tangent stiffness matrix $\partial \sigma / \partial \varepsilon$ at each integration point to finally obtain equilibrium at the global scale. For obtaining the thermal strains as well as the yield stress $\sigma_{y}$ by means of the KWN model, it is necessary to calculate the temperature $T$. This is done by calling a subroutine that solves the transient heat equation by means of Green's functions. The KWN model is temporally integrated by an implicit Euler scheme. As outlined in Section 5, the evolution of the KWN model is described by a growth law and a nucleation rate that show both a strong temperature dependency. Because of the spatial variation of the microstructure the complete discrete particle size distribution must be stored at each integration point for describing the microstructure evolution. Due to the knowledge of the particle size distribution, it is possible to calculate the macroscopic yield strength for the material model. The great advantage of describing the microstructure via a KWN model compared to a purely phenomenological model is that recalibration of the welding zone and the HAZ is not necessary. Furthermore, the physical description allows a deeper understanding of the process and enables the development of improved simulation models.

\subsection{Investigated material}

The aluminium-magnesium-silicon alloy AA6082 in T6 temper condition is investigated. AA6082 is widely used in industry for various applications. The chemical composition of the investigated alloy is given in Table 1 and was determined by spark spectrometry. The widely accepted precipitation sequence of aluminium magnesium silicon alloys is: SSSS (Supersaturated solid solution) $\rightarrow$ GP zones $\rightarrow \beta^{\prime \prime} \rightarrow \beta^{\prime} / B^{\prime} \rightarrow \beta$ (stable), see e.g. Edwards et al. (1998) and Marioara et al. (2001).

Table 1: Nominal chemical composition of the investigated aluminium sheets in wt- $\%$.

\begin{tabular}{|l|l|l|l|l|l|l|}
\hline$C_{M g}^{0}$ & $C_{S i}^{0}$ & $C_{C u}^{0}$ & $C_{M n}^{0}$ & $C_{F e}^{0}$ & Other & $C_{A l}^{0}$ \\
\hline 0.905 & 1.195 & 0.087 & 0.444 & 0.361 & 0.113 & balance \\
\hline
\end{tabular}




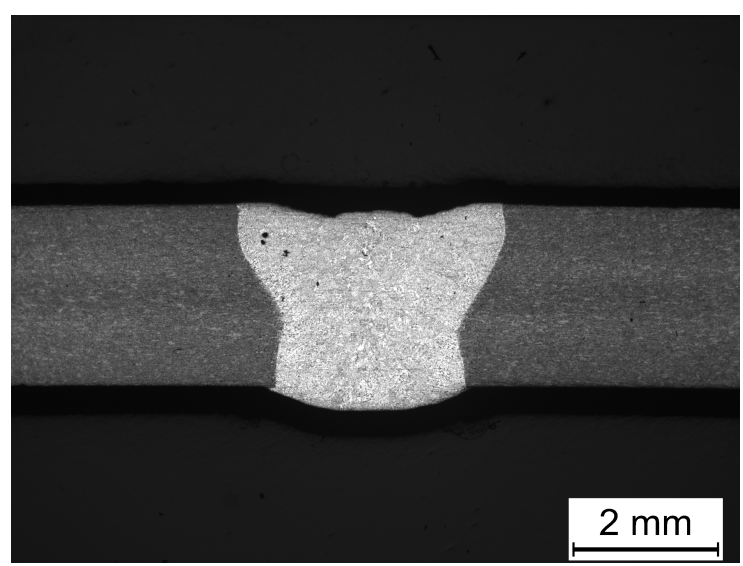

(a)

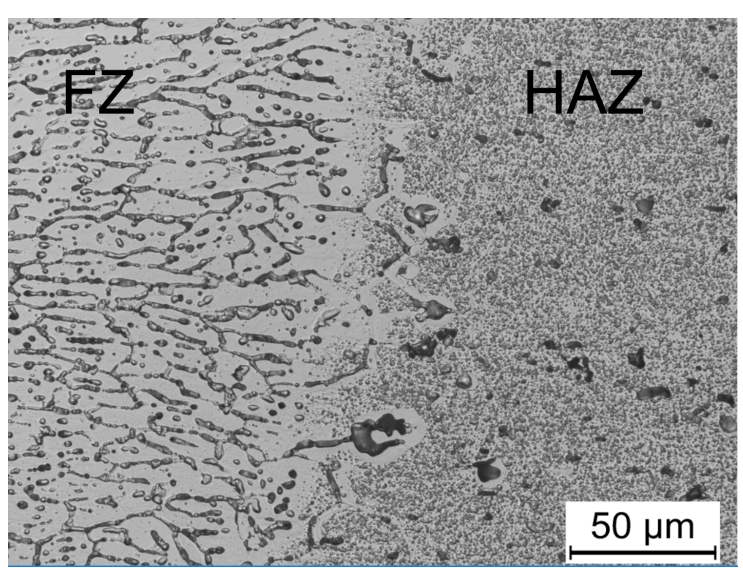

(b)

Figure 2: (a) Etched micrograph of laser beam welded AA6082-T6. (b) Micrograph showing fusion zone (FZ) and HAZ.

\subsection{Laser beam welding process}

The laser beam welding process was carried out by a continuous wave ytterbium fibre laser YLS-8000-S2-Y12 (IPG Photonics Corporation) with a maximum laser power of $8 \mathrm{~kW}$. The laser was connected to an YW52 Precitec optical head, which was mounted onto a CNC-supported machining centre (IXION Corporation PLC). AA6082-T6 aluminium sheets of $2.5 \mathrm{~mm}$ thickness were welded bead on plate without filler wire. The sheets have a length of $100 \mathrm{~mm}$ and a width of $200 \mathrm{~mm}$. A constant laser power of $4 \mathrm{~kW}$ and a welding speed of $2 \mathrm{~m} / \mathrm{min}$ were used. The welding was performed in a gas shielding box, which was floated with argon. The weld seam has a wide Y-shape according to Fig.2(a). The resulting width of the fusion zone at the radiation exposure site is approximately $3.6 \mathrm{~mm}$. The width of the weld seam root is $2.6 \mathrm{~mm}$.

\subsection{Temperature measurement}

In our study we used a thermal camera (Optris PI 420) for measuring the temperature during the welding process. In order to increase the emission of the substrate surface and thus to enhance the credibility of the thermography measurements, the measured surface in the HAZ were coated with temperature resistant black paint. The approximate resolution of the thermal camera under the given experimental setup was $0.4 \mathrm{~mm}$.

\subsection{Hardness measurement}

The hardness measurements were performed with a HMV-2000 Hardness Tester from Shimadzu. The indentations have been arranged in a line that was located approximately in the centre of the micrograph of Fig.2(a). The hardness measurements were performed roughly 24 hours after welding. The test force corresponded to HV0.2 and the distance between adjacent hardness indentations was $0.44 \mathrm{~mm}$.

\subsection{Residual stress analysis via X-ray diffraction}

X-ray diffraction was performed at the HZG beamline P07B located at PETRA III at DESY (Deutsches Elektronen Synchrotron, Hamburg, Germany). The measurements were carried out in transmission in Debye-Scherrer geometry at a photon energy of $87.1 \mathrm{keV}$, corresponding to a wavelength of $0.1423 \AA$. Thus, no information on stress gradients along the sheet thickness was obtained. A Perkin-Elmer detector with an active area of $400 \mathrm{~mm} \times 400 \mathrm{~mm}$ and a pixel size of $200 \mu \mathrm{m}$ was used for recording complete diffraction rings. The distance between sample and detector was $1526 \mathrm{~mm}$. Cu powder paste was applied to the sheet for calibrating the distance between specimen and detector. The Al (311) reflection was used to determine the strain. The diffraction images were reduced to diffraction patterns by azimuthal integration within different sectors using the program Fit2d, see Hammersley et al. (1996, 1997). The 
sectors had a size of $10^{\circ}$. The mean values of the sectors at $0^{\circ}$ and $180^{\circ}$ and the sectors at $90^{\circ}$ and $270^{\circ}$ were used for determining strains in longitudinal and transverse directions, respectively.

Single scan lines across the weld were measured for several sheets. Each line was scanned with $0.5 \mathrm{~mm} \times 0.5 \mathrm{~mm}$ beam cross-section and at a $0.5 \mathrm{~mm}$ step size. An area of size $196 \mathrm{~mm} \times 30 \mathrm{~mm}$ was measured for one sheet. The area scans consist of 1200 diffraction patterns obtained with $0.5 \mathrm{~mm} \times 0.5 \mathrm{~mm}$ beam cross-section and a $0.5 \mathrm{~mm}$ step size in longitudinal direction. The strain $\varepsilon$ was calculated from the diffraction patterns as

$$
\varepsilon=\frac{\Delta d}{d_{0}}=-\Delta \theta \cot \left(\theta_{0}\right)
$$

where $\Delta \theta$ and $\Delta d$ are changes in the Bragg angle and lattice spacing respectively, from their corresponding values $\theta_{0}$, $d_{0}$ of the stress-free sample. This equation holds for every considered direction. To derive stresses from the strain, the following equations of linear elasticity were used where it is assumed that the sample is thin enough so that plane stress conditions apply $\left(\sigma_{z z}=0\right)$ :

$$
\sigma_{x x}=E \frac{\varepsilon_{y y}+v \varepsilon_{x x}}{1-v^{2}}, \quad \sigma_{y y}=E \frac{\varepsilon_{x x}+v \varepsilon_{y y}}{1-v^{2}} .
$$

The stress-free Bragg angle $\theta_{0}$ was determined from a reference sample that was cut across the weld line by electrodischarge machining. The reference sample had a size of $2 \mathrm{~mm} \times 64 \mathrm{~mm} \times 2.5 \mathrm{~mm}$, where $2.5 \mathrm{~mm}$ is the thickness of the sheet and $64 \mathrm{~mm}$ is the size in transverse direction. The macro-stresses can relax during cutting of such a thin sample. The reference was considered representative for the weld within the analysed area. $\mathrm{Cu}$ powder paste was used for calibration of the reference sample. The $\mathrm{Al}$ (311) reflection was chosen as the one that is least affected by microstrain (Fitzpatrick and Lodini, 2003) to determine macro-stresses. In Hooke's law, Young's modulus $E=70 \mathrm{GPa}$ and Poisson's ratio $v=0.3$ for aluminium bulk were used during the calculations. The deviation of the X-ray elastic constants of the (310) reflection from the bulk values are small.

\section{Temperature field}

\subsection{Method of Green's Functions}

A very general method for solving the linear heat conduction equation is the method of Green's functions. The following section summarizes the basic ideas of this method. For a general overview and the complete discussion of various types of boundary conditions, the reader is referred to Beck et al. (1992).

A general domain $\Omega$ with boundary $\Gamma$ is assumed. A point in $\Omega$ is denoted with $\mathbf{x}$. For simplicity, the boundary is composed of boundaries on which a temperature or heat flux are defined. The boundaries of prescribed temperature are denoted with $\Gamma_{T}$. Respectively, the boundaries with prescribed heat flux are described by $\Gamma_{q}$. The boundary is completely defined by these two types of boundary conditions; therefore, it holds $\Gamma_{T} \cup \Gamma_{q}=\Gamma$. The heat source is denoted by $g: \Omega \times] 0, t[\rightarrow \mathbb{R}$. The initial temperature condition of the structure is defined by $F: \Omega \rightarrow \mathbb{R}$. The temperature field $T: \Omega \times[0, t] \rightarrow \mathbb{R}$ must fulfil the heat equation with boundary conditions as follows

$$
\begin{array}{llll}
\nabla^{2} T+\frac{1}{k} g=\frac{1}{\alpha} \frac{\partial T}{\partial t} & \text { for } & \Omega \times] 0, t[, \\
T & =f_{T} & \text { on } & \left.\Gamma_{T} \times\right] 0, t[, \\
k \frac{\partial T}{\partial \mathbf{n}} & =f_{q} & \text { on } & \left.\Gamma_{q} \times\right] 0, t[, \\
T(\mathbf{x}, 0) & =F . &
\end{array}
$$

The thermal conductivity $k$, thermal diffusivity $\alpha$ and heat transfer coefficient $h$ are assumed to be constant. The method of Green's functions now transfers the boundary value problem into an integral equation that can be solved by integration, if a solution of the so called auxiliary boundary value problem is available, see Beck et al. (1992). The solution of that auxiliary problem is the Green's function $G\left(\mathbf{x}, t \mid \mathbf{x}^{\prime}, \tau\right)$. 
Following the derivation given by Beck et al. (1992), the temperature field is described by contributions from the initial conditions, boundary conditions and heat sources

$$
T(\mathbf{x}, t)=T_{I C}(\mathbf{x}, t)+T_{B C}(\mathbf{x}, t)+T_{H S}(\mathbf{x}, t) .
$$

The terms $T_{I C}$ and $T_{H S}$ take into account the influences from the initial conditions and the heat source term

$$
T_{I C}(\mathbf{x}, t)=\int_{\Omega} G\left(\mathbf{x}, t \mid \mathbf{x}^{\prime}, 0\right) F\left(\mathbf{x}^{\prime}\right) d \mathbf{x}^{\prime}, \quad T_{H S}(\mathbf{x}, t)=\frac{\alpha}{k} \int_{\tau=0}^{t} \int_{\Omega} G(\mathbf{x}, t \mid \dot{\mathbf{x}}, \tau) g(\hat{\mathbf{x}}, \tau) d \mathbf{x}^{\prime} d \tau .
$$

The contribution from the boundary conditions is described by

$$
T_{B C}(\mathbf{x}, t)=-\left.\alpha \int_{0}^{t} \int_{\Gamma_{T}} f_{T}\left(\mathbf{x}^{\prime}, \tau\right) \frac{\partial G\left(\mathbf{x}, t \mid \mathbf{x}^{\prime}, \tau\right)}{\partial \mathbf{n}^{\prime}}\right|_{\mathbf{x}^{\prime}=\Gamma_{T}} d \Gamma^{\prime} d \tau+\frac{\alpha}{k} \int_{0}^{t} \int_{\Gamma_{q}} f_{q}\left(\mathbf{x}^{\prime}, \tau\right) G\left(\mathbf{x}, t \mid \mathbf{x}^{\prime}, \tau\right) d \Gamma^{\prime} d \tau .
$$

The essential prerequisite for the usage of the method of Green's function is the availability of the suitable Green's function, which depends on the considered geometry as well as the prescribed type of boundary condition.

\subsection{Formulation of the boundary value problem}

The solution of partial differential equations by means of Green's functions requires the knowledge of the specific Green's function for the considered geometry and boundary conditions. The very general solution presented in Section 3.1 can be simplified considerably, if adiabatic boundary conditions in combination with homogeneous prescribed temperature boundary conditions and a homogeneous initial condition are considered. These assumptions are expressed by

$$
\left.k \frac{\partial T}{\partial \mathbf{n}}\right|_{\Gamma_{q}}=0,\left.\quad T\right|_{\Gamma_{T}}=T_{0}, \quad T(\mathbf{x}, 0)=T_{0} \quad \text { in } \quad \Omega,
$$

where the same temperature $T_{0}$ is assumed as boundary as well as initial condition. The solution of the heat equation is now further simplified for the considered boundary conditions of Eq.(3.10). The boundary and initial conditions can be homogenized by a substitution $T(\mathbf{x}, t)=\Psi(\mathbf{x}, t)+T_{0}$, introducing an auxiliary function $\Psi(\mathbf{x}, t)$. Due to this substitution, the integrals given in $T_{I C}$ and $T_{B C}$, according to Eq.(3.8) and Eq.(3.9), are vanishing and only the term $T_{H S}$ must be considered further. After back substitution, the following expression for the calculation of the temperature $T$ remains

$$
T(\mathbf{x}, t)=\frac{\alpha}{k} \int_{\tau=0}^{t} \int_{\Omega} G(\mathbf{x}, t \mid \dot{\mathbf{x}}, \tau) g(\hat{\mathbf{x}}, \tau) d \mathbf{x}^{\prime} d \tau+T_{0} .
$$

For investigation of a butt welding process, it is now necessary to define the geometry and the boundary conditions for setting up the corresponding Green's function of the investigated problem. The relevant geometry is shown in Fig.3. The welded sheet geometry is defined by $\Omega:=\left(0, L_{x}\right) \times\left(0, L_{y}\right) \times\left(0, L_{z}\right)$. For Cartesian coordinates, the Green's function, which fulfils the auxiliary boundary value problem, can be assembled as a product of one dimensional Green's functions. Hence, Green's function for the heat equation in one dimension are introduced briefly. Following Beck et al. (1992) the Green's function for prescribed temperature boundary conditions at both boundaries is denoted by a (11)-subscript. Accordingly, boundary conditions of prescribed heat flux at both boundaries is indicated by a (22)-subscript. As an example, the Green's function for adiabatic boundary conditions in the x-y and y-z plane as well as prescribed temperature in the $\mathrm{x}-\mathrm{z}$-plane for the considered geometry $\Omega$ reads

$$
G(\mathbf{x}, t \mid \mathbf{x}, \tau)=G_{22}\left(x, t \mid x^{\prime}, \tau\right) G_{22}\left(z, t \mid z^{\prime}, \tau\right) G_{11}\left(y, t \mid y^{\prime}, \tau\right)
$$

using the one dimensional Green's function from Beck et al. (1992) for boundary conditions of first type:

$$
G_{11}\left(x, t \mid x^{\prime}, \tau\right)=\frac{1}{\sqrt{4 \pi \alpha[t-\tau]}} \sum_{n=-\infty}^{\infty}\left[\exp \left(-\frac{\left[2 n L+x-x^{\prime}\right]^{2}}{4 \alpha[t-\tau]}\right)-\exp \left(-\frac{\left[2 n L+x+x^{\prime}\right]^{2}}{4 \alpha[t-\tau]}\right)\right]
$$

and second type:

$$
G_{22}\left(x, t \mid x^{\prime}, \tau\right)=\frac{1}{\sqrt{4 \pi \alpha[t-\tau]}} \sum_{n=-\infty}^{\infty}\left[\exp \left(-\frac{\left[2 n L+x-x^{\prime}\right]^{2}}{4 \alpha[t-\tau]}\right)+\exp \left(-\frac{\left[2 n L+x+x^{\prime}\right]^{2}}{4 \alpha[t-\tau]}\right)\right] .
$$




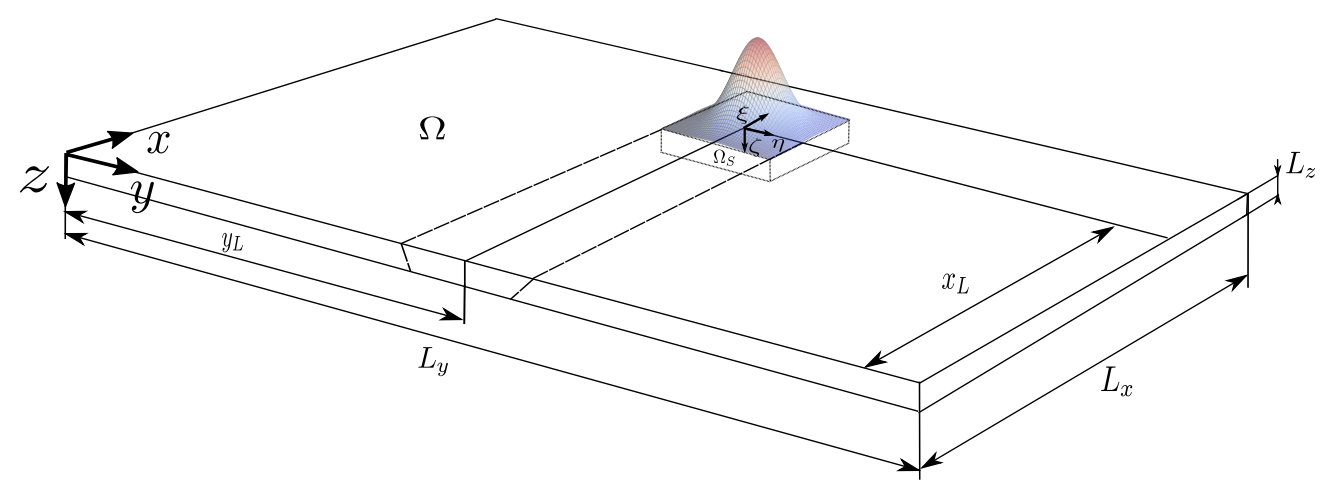

Figure 3: Schematic presentation of the investigated domain $\Omega$ including its dimensions. The time dependent domain of the source is denoted by $\Omega_{s}$. The figure shows the heat source for constant $\mathrm{z}=0$ and the introduced moving coordinate system that is attached to the centre of the heat source.

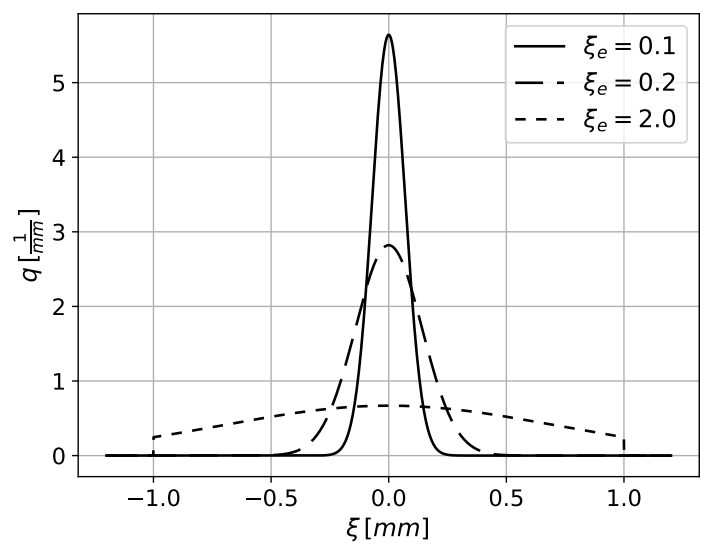

(a)

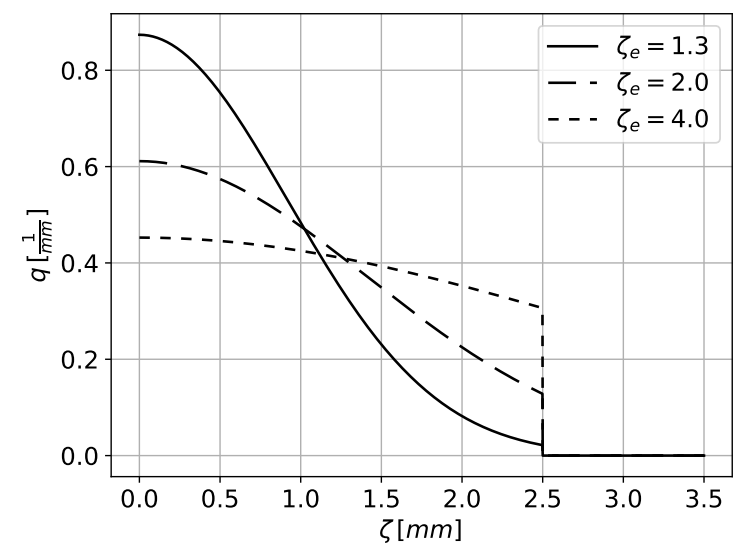

(b)

Figure 4: Parameter variation for one dimensional Gaussian distribution according Eq.(3.15). (a) Exemplary distribution with bounds of $\xi^{\prime}=$ $-1.0 \mathrm{~mm}$ and $\xi^{\prime \prime}=1.0 \mathrm{~mm}$. (b) Exemplary distribution in plate thickness direction $\zeta^{\prime}=0 \mathrm{~m}$ and $\zeta^{\prime \prime}=2.5 \mathrm{~mm}$.

\subsection{Formulation of the heat source model}

To obtain analytic solutions for the discussed boundary conditions, it is necessary to define suitable heat sources. In this paper, a Gaussian distribution is investigated. For definition of heat sources, it is useful to introduce a relative coordinate system with coordinates $(\xi, \eta, \zeta)$, which is attached to the center of the heat source according to Fig. 3. In a first step, a one dimensional heat source is described. The following one-dimensional heat source $f_{\chi}: \chi \rightarrow \mathbb{R}, \chi \in$ $\left[\chi^{\prime}, \chi^{\prime \prime}\right]$ has been proposed by Karkhin et al. (2011)

$$
f_{\chi}(\chi)=\frac{2}{\sqrt{\pi} \chi_{e}\left[\operatorname{erf}\left(\frac{\chi^{\prime \prime}}{\chi_{e}}\right)-\operatorname{erf}\left(\frac{\chi^{\prime}}{\chi_{e}}\right)\right]} e^{-\left(\frac{\chi}{\chi_{e}}\right)^{2}}
$$

with $\chi=\{\xi, \eta, \zeta\}$ for every room direction. The integration between the upper limit $\chi^{\prime \prime}$ and lower limit $\chi^{\prime}$ of the heat source yields $\int_{\chi^{\prime}}^{\chi^{\prime \prime}} f_{\mathcal{X}}(\chi)=1$. The parameter $\chi_{e}$ has to be determined by adapting the simulation results to experimental data. Fig.4 illustrates the influence of the considered interval limits and different parameters. For now, it is convenient to limit the discussion to the $\xi$-direction. The generalization to three dimensions is then evident. The center of the Gaussian heat source is denoted by $x_{L}$. The local coordinate is denoted by $\xi$ and the relationship between the local coordinate system and the global coordinate system is simply $x=\xi+x_{L}(t)$. The description of the heat source according to Eq.(3.15) in global coordinates must take into account the limits of the heat source by using the Heaviside 
function $H(\bullet)$ which leads to

$$
\bar{f}_{\xi}(x, t)=\frac{2\left[H\left(x-\left[\xi^{\prime}+x_{L}\right]\right)-H\left(x-\left[\xi^{\prime \prime}+x_{L}\right]\right)\right]}{\sqrt{\pi} \xi_{e}\left[\operatorname{erf}\left(\frac{\xi^{\prime \prime}}{\xi_{e}}\right)-\operatorname{erf}\left(\frac{\xi^{\prime}}{\xi_{e}}\right)\right]} e^{-\left(\frac{x-x_{L}}{\xi_{e}}\right)^{2}} .
$$

A three-dimensional temperature distribution $g$ can be constructed by multiplication of the one-dimensional normally distributed heat source according to Eq.(3.15), in every spatial direction, formulated in the local coordinates $(\xi, \eta, \zeta)$ and weighted by the absorbed welding power $Q$

$$
g(\xi, \eta, \zeta)=Q f_{\xi}(\xi) f_{\eta}(\eta) f_{\zeta}(\zeta)
$$

This heat source is active in the domain $\Omega_{S}$, as indicated in Fig.3.

\subsection{Integration of Green's function}

The solution of the heat equation subjected to a moving heat source according to Eq.(3.17) and boundary conditions as discussed for the Green's function according to Eq.(3.12) for the domain $\Omega$, is completely determined by Eq.(3.11). The insertion of the Green's function $G$ given by Eq.(3.12) and the heat source $g$ according to Eq.(3.17) into the integral Eq.(3.11) and using the properties of the Heaviside function leads to the following integral

$$
\begin{aligned}
T(\mathbf{x}, t)=\frac{\alpha Q}{k} \int_{\tau=0}^{t_{w}}[ & \int_{\xi^{\prime}}^{\xi^{\prime \prime}} G_{22}\left(x, t \mid \xi+x_{L}, \tau\right) \bar{f}_{\xi}\left(\xi+x_{L}, \tau\right) d \xi \\
& \int_{\eta^{\prime}}^{\eta^{\prime \prime}} G_{11}\left(y, t \mid \eta+y_{L}, \tau\right) \bar{f}_{\eta}\left(\eta+y_{L}, \tau\right) d \eta \\
& \left.\int_{\zeta^{\prime}}^{\zeta^{\prime \prime}} G_{22}\left(z, t \mid \zeta+z_{L}, \tau\right) \bar{f}_{\zeta}\left(\zeta+z_{L}, \tau\right) d \zeta\right] d \tau+T_{0} .
\end{aligned}
$$

The total time of the active heat source is denoted by $t_{w}$. It is obvious that every direction can be treated separately. The integration of the product resulting from one dimensional Green's function and the heat source can be performed by analytic integration. For this purpose, the following notation is introduced

$$
F_{22}(x, t, \tau)=\int_{\xi^{\prime}}^{\xi^{\prime \prime}} G_{22}\left(x, t \mid \xi+x_{L}, \tau\right) f_{\xi}\left(\xi+x_{L}, \tau\right) d \xi=\Im_{22}\left(x, t, \xi^{\prime \prime}, \tau\right)-\Im_{22}\left(x, t, \xi^{\prime}, \tau\right)
$$

where the function $\mathfrak{\mho}_{22}$ is composed via

$$
\begin{aligned}
x_{L} & =x_{0}+v_{x} \tau \\
k_{n} & =2 n L_{x}+x, \\
k_{\tau} & =4 \alpha[t-\tau], \\
c_{e f} & =\sqrt{k_{\tau}\left[\xi_{e}^{2}+k_{\tau}\right]} \xi_{e} \\
c_{2} & =\xi_{e}^{2}\left[x_{L}+\xi\right]+k_{\tau} \xi \\
\mathfrak{J}_{22 / 11}(x, t, \xi, \tau)^{1} & =\frac{1}{\sqrt{\pi}\left[\operatorname{erf}\left(\frac{\xi^{\prime \prime}}{\xi_{e}}\right)-\operatorname{erf}\left(\frac{\xi^{\prime}}{\xi_{e}}\right)\right]} \frac{1}{\sqrt{\xi_{e}^{2}+k_{\tau}}} \sum_{n=-\infty}^{\infty}\left[ \pm \exp \left(\frac{-\left[k_{n}+x_{L}\right]^{2}}{\xi_{e}^{2}+k_{\tau}}\right) \operatorname{erf}\left(\frac{k_{n} \xi_{e}^{2}+c_{2}}{c_{e f}}\right)\right. \\
& \left.+\exp \left(\frac{-\left[k_{n}-x_{L}\right]^{2}}{\xi_{e}^{2}+k_{\tau}}\right) \operatorname{erf}\left(\frac{-k_{n} \xi_{e}^{2}+c_{2}}{c_{e f}}\right)\right] .
\end{aligned}
$$

assuming a movement of the x-component of the centre of the heat source $x_{L}$, starting from point $x_{0}$ with a velocity $v_{x}$ in $x$-direction. Here, $\mathfrak{F}_{22}$ denotes the antiderivative of the product of the one dimensional Green's function for adiabatic

\footnotetext{
${ }^{1} \widetilde{F}_{11}$ denotes the result of the integration of Eq.(3.19) by replacing the Green's function $G_{22}$ by $G_{11}$.
} 
boundary conditions $G_{22}$ and the heat source function $f_{\xi}$ corresponds to the first inner integral of Eq.(3.18). The similar integration procedure delivers for integration of a product of one dimensional Green's function for boundary conditions of the first kind $G_{11}$ and the heat source function $f_{\xi}$, the antiderivative $\mathfrak{\Im}_{11}$, using the minus sign in Eq.(3.20). The exchange of variables for the different room directions is obvious. The last remaining integral over time is solved by Gauss-Conrod-quadrature, see Piessens et al. (2012). The implementation of the model is summarized in Algorithm 1 and the used parameters in the temperature model are provided in Table 2. It is evident that the numerical effort is significantly reduced by this procedure compared to the commonly used numerical methods for the solution of partial differential equations.

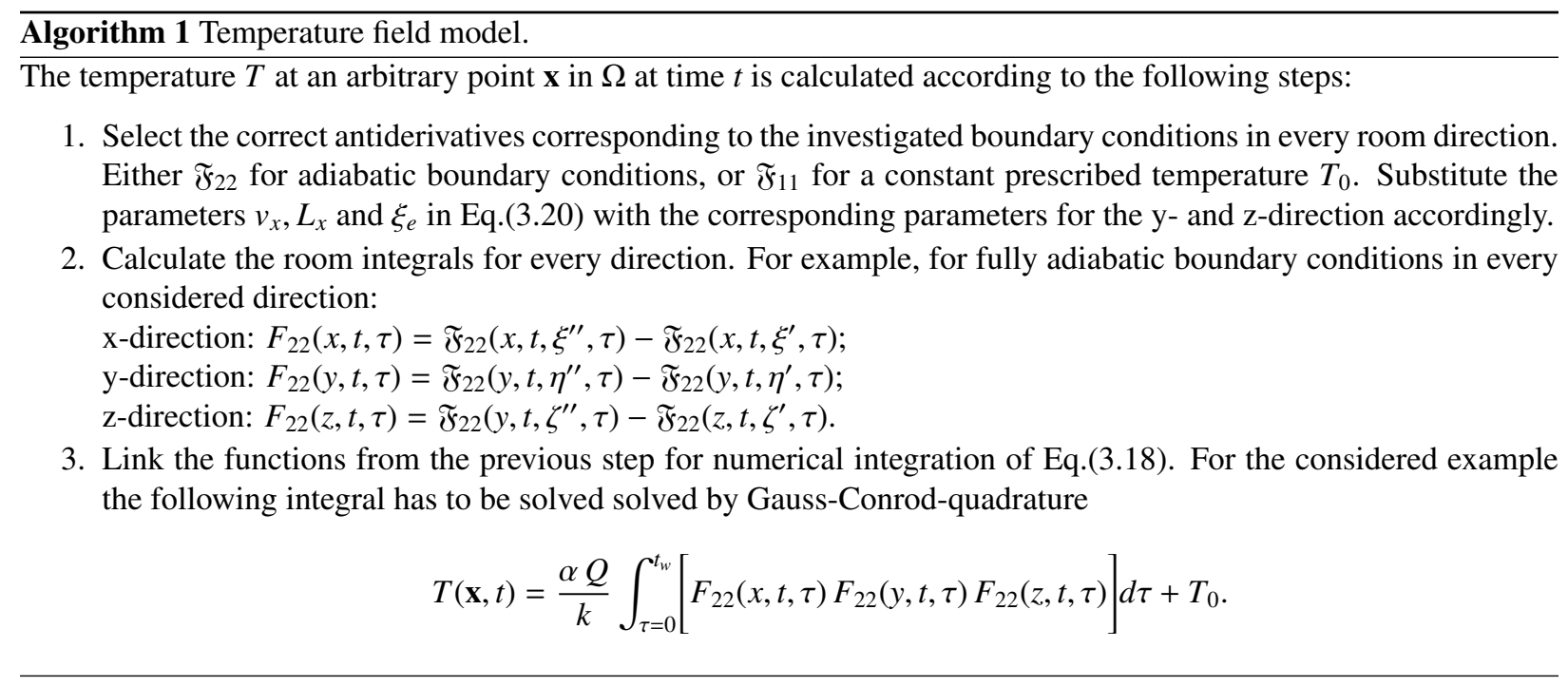

\section{Material model}

\section{Modelling strategy}

The constitutive modelling of plasticity phenomena, where the effect of phase changes is taken into account, is established for casting processes, e.g. Cervera et al. (1999). Since the laser beam welding process can be interpreted as a casting process with exceptional high cooling rates, this modelling approach is also applicable to welding and additive manufacturing processes, as shown by Chiumenti et al. (2010). The following material model incorporates the modelling strategy by Chiumenti et al. (2010) and relates the evolution of stresses and plastic strains to the underlying microstructure by means of a KWN model.

In welding processes, only small deformations are present after solidification. Therefore, a small strain theory is chosen for simplicity. It is assumed that the strain $\boldsymbol{\varepsilon}$ is fully described by thermal strains $\boldsymbol{\varepsilon}^{\Theta}$, elastic strains $\boldsymbol{\varepsilon}^{e}$ and plastic strains $\boldsymbol{\varepsilon}^{p}$. Further, a von-Mises flow potential is used under usage of the deviator $\mathbf{s}=\operatorname{dev}(\boldsymbol{\sigma})$. A temperature dependent solid fraction $f_{s}$ is used, which scales the current yield stress $\sigma_{y}$ leading to

$$
\boldsymbol{\varepsilon}=\boldsymbol{\varepsilon}^{e}+\boldsymbol{\varepsilon}^{\Theta}+\boldsymbol{\varepsilon}^{p}, \quad \Phi=\sqrt{\frac{3}{2} \mathbf{s}: \mathbf{s}}-\sigma_{y} f_{s} \leq 0, \quad f^{s} \in[0,1] .
$$

The solid fraction $f_{s}$ is used to describe the material behaviour in the mushy zone. An associative flow rule in conjunction with a von-Mises flow criteria is used to determine the evolution of plastic strains $\boldsymbol{\varepsilon}^{p}$. The thermoelasto-viscoplastic material behaviour is achieved under usage of the the viscosity parameter $\eta$ leading to a modified Bingham-model:

$$
\dot{\boldsymbol{\varepsilon}}^{p}=\dot{\gamma} \frac{\partial \Phi}{\partial \mathbf{s}}=\dot{\gamma} \sqrt{\frac{3}{2}} \frac{\mathbf{s}}{\|\mathbf{s}\|}, \quad \dot{\gamma}=\frac{1}{\eta}\left\langle\sqrt{\frac{3}{2} \mathbf{s}: \mathbf{s}}-\sigma_{y} f^{s}\right\rangle .
$$


Table 2: Parameters used in calculation of the temperature field, see Section 3, based on the method of Green's functions.

\begin{tabular}{|l|l|l|l|}
\hline Parameter & Symbol & Value & Unit \\
\hline Specimen length & $L_{x}$ & 100 & $\mathrm{~mm}$ \\
Specimen width & $L_{y}$ & 200 & $\mathrm{~mm}$ \\
Specimen thickness & $L_{z}$ & 2.5 & $\mathrm{~mm}$ \\
Welding speed & $v_{x}$ & 2 & $\mathrm{~m} / \mathrm{min}$ \\
Welding power & $Q$ & 1684 & $\mathrm{~W}$ \\
Shape parameter for x & $\xi_{e}$ & 0.001 & $\mathrm{~mm}$ \\
Shape parameter for y & $\eta_{e}$ & 0.001 & $\mathrm{~mm}$ \\
Shape parameter for z & $\zeta_{e}$ & 1.3 & $\mathrm{~mm}$ \\
Upper source bound in x & $\xi^{\prime \prime}$ & 0.5 & $\mathrm{~mm}$ \\
Lower source bound in x & $\xi^{\prime}$ & -0.5 & $\mathrm{~mm}$ \\
Upper source bound in y & $\eta^{\prime \prime}$ & 0.5 & $\mathrm{~mm}$ \\
Lower source bound in y & $\eta^{\prime}$ & -0.5 & $\mathrm{~mm}$ \\
Upper source bound in $\mathrm{z}$ & $\zeta^{\prime \prime}$ & 0 & $\mathrm{~mm}$ \\
Lower source bound in $\mathrm{z}$ & $\zeta^{\prime}$ & 2.5 & $\mathrm{~mm}$ \\
Width of the source & $\xi^{\prime}$ & -0.5 & $\mathrm{~mm}$ \\
Thermal conductivity & $\mathrm{k}$ & 170 & $\mathrm{w} / \mathrm{mK}$ \\
Heat capacity & $c_{p}$ & 896 & $\mathrm{~J} / \mathrm{kgK}$ \\
\hline
\end{tabular}

The Macaulay brackets $\langle\bullet\rangle$ are used for convenience. The elastic and thermal strains are suppressed in the fusion and mushy zone via a modified Hook's law, which scales the bulk modulus $K$ and shear modulus $G$ as follows

$$
\sigma=\frac{K}{f^{s}} \varepsilon_{v}^{e} \mathbf{I}+2 \frac{G}{f^{s}} \operatorname{dev}\left(\boldsymbol{\varepsilon}^{e}\right)
$$

I represents the second order identity tensor. The material model formulation shows thermo-elasto-viscoplastic material behaviour in the solid case $\left(f_{s}=1\right)$ and pure viscous behaviour in the liquid case $\left(f_{s}=0\right)$. Taking into account the definition of the plastic multiplier in Eq.(4.22), the following dependency is obvious

$$
\dot{\boldsymbol{\varepsilon}}^{p}=\dot{\gamma} \sqrt{\frac{3}{2}} \frac{\mathbf{s}}{\|\mathbf{s}\|}=\frac{3}{2} \frac{1}{\eta} \mathbf{s}, \quad \boldsymbol{\varepsilon}^{e}=0, \quad \varepsilon_{v}=\text { const., } \quad \text { if } \quad f^{s}=0
$$

The phase change from liquid to solid in aluminium is finished when the solid face-centred-cubic aluminium crystalic structure has developed. The evolution equation for development of the solid fraction $f_{s}$ in the temperature range between the liquidus temperature $T_{L}$ and the solidus temperature $T_{S}$ is based on a derivation given in Chiumenti et al. (2010). There, it is assumed that solidification occurs without diffusion in the solid phase. Further, the equilibrium composition between solid and liquid phase should be rather constant over the range of solidification. According to Chiumenti et al. (2010) the unregularized solid fraction is

$$
\bar{f}_{S}(T)=1-\left[\frac{T_{F}-T}{T_{F}-T_{L}}\right]^{\frac{1}{k-1}} \quad \text { with } \quad k=\frac{T_{F}-T_{L}}{T_{F}-T_{S}} .
$$

Here, $\mathrm{k}$ is the partition coefficient. The solidification temperature of the pure component is denoted with $T_{L}$. For the solidus temperature $T_{S}$, Eq.(4.25) shows an instantaneous solidification, which can be regularized by a regularization 
temperature $\Delta T$ according to Chiumenti et al. (2010)

$$
f_{s}(T)=\left\{\begin{array}{lll}
1-\left[\frac{T_{F}-T}{T_{F}-T_{L}}\right]^{\frac{1}{k-1}} & \text { for } & T_{S}+\Delta T \leq T \leq T_{L} \\
{\left[1-\bar{f}_{S}\left(T_{S}+\Delta T\right)\right]\left[\frac{T_{S}-T}{\Delta T}\right]} & \text { for } & T_{S} \leq T \leq T_{S}+\Delta T
\end{array}\right.
$$

The fully implicit elastic return-mapping algorithm with nonlinear isotropic hardening for the material has been outlined by Herrnring and Klusemann (2017) and summarized in Algorithm 2 for completeness. Due to the incompressible material behaviour in the liquid case, a mixed $\mathrm{u} / \mathrm{p}$-finite-element formulation is used. The finite element calculation of residual stresses is preformed in the finite element software ABAQUS. The material routine is implemented as a user material (UMAT) subroutine.

Algorithm 2 Integration of elasto-visco-plastic material model for welding processes over time interval $\left[t_{n}, t_{n+1}\right]$. Extended algorithm based on Herrnring and Klusemann (2017).

The step at $t_{n}$ with strains $\left\{\boldsymbol{\varepsilon}_{n}, \boldsymbol{\varepsilon}_{n}^{p}, \varepsilon_{n}^{p}\right\}$ and the strain increment $\Delta \boldsymbol{\varepsilon}$ are given.

1. Calculate $T_{n+1}, f_{n+1}^{s}, \boldsymbol{\varepsilon}_{n+1}^{\Theta}$. Modify material constants in Hook's law: $G=\frac{G}{f_{n+1}^{s}}$ and $K=\frac{K}{f_{n+1}^{s}}$.

2. Calculate the time increment in the KWN model and update internal variables as outlined in Section 5.

3. Make a trial step: $\boldsymbol{\varepsilon}_{n+1}^{\text {etrial }}=\boldsymbol{\varepsilon}_{n}+\Delta \boldsymbol{\varepsilon}-\boldsymbol{\varepsilon}_{n+1}^{\Theta}-\boldsymbol{\varepsilon}_{n}^{p}, \quad \varepsilon_{v n+1}^{\text {etrial }}=\operatorname{trace}\left(\boldsymbol{\varepsilon}_{n+1}^{\text {etrial }}\right), \quad \boldsymbol{\varepsilon}_{d n+1}^{\text {etrial }}=\boldsymbol{\varepsilon}_{n+1}^{\text {etrial }}-\frac{1}{3} \varepsilon_{v n+1}^{\text {etrial }} \mathbf{I}$, $p_{n+1}^{\text {trial }}=K \varepsilon_{v n+1}^{\text {etrial }}, \quad \boldsymbol{s}_{n+1}^{\text {trial }}=2 G \varepsilon_{d n+1}^{\text {etrial }}, \quad \varepsilon_{n+1}^{\text {ptrial }}=\varepsilon_{n}^{p}, \quad \varepsilon_{n+1}^{p \text { trial }}=\varepsilon_{n}^{p}, \quad q_{n+1}^{\text {trial }}=\sqrt{\frac{3}{2} s_{n+1}^{\text {trial }}: s_{n+1}^{\text {trial }} .}$

4. Check the flow condition: If $q_{n+1}^{\text {trial }}-f_{n+1}^{s} \sigma_{y}\left(\varepsilon_{n+1}^{\text {ptrial }}\right) \leq 0$ then $(\cdot)_{n+1}=(\cdot)_{n+1}^{\text {trial }}$ and calculate $\mathbb{C}=2 G \mathbb{I}_{d}+K \mathbf{I} \otimes \mathbf{I} . \mathbb{I}_{d}$ is calculated as defined in step 6. Leave the material routine.

5. Solve the nonlinear equation $\tilde{\Phi}(\Delta \gamma)$ for $\Delta \gamma$ : $\tilde{\Phi}(\Delta \gamma)=\Delta \gamma-\frac{\Delta t}{\eta}\left[q_{n+1}^{t r i a l}-3 G \Delta \gamma-f_{n+1}^{s} \sigma_{y}\left(\varepsilon_{n+1}^{p}\right)\right]=0$

6. Determine stresses, strains and tangent stiffness matrix $\mathbb{C}$

$$
\begin{aligned}
& p_{n+1}=p_{n+1}^{\text {trial }}, \quad \mathbf{s}_{n+1}=\left[1-\frac{3 \Delta \gamma G}{q_{n+1}^{\text {trial }}}\right] \mathbf{s}_{n+1}^{\text {trial }}, \quad \boldsymbol{\sigma}_{n+1}=\mathbf{s}_{n+1}+p_{n+1} \mathbf{I}, \quad \boldsymbol{\varepsilon}_{n+1}^{p}=\boldsymbol{\varepsilon}_{n}^{p}+\Delta \gamma \sqrt{\frac{3}{2}} \frac{\mathbf{s}_{n+1}}{\left\|\mathbf{s}_{n+1}\right\|}, \\
& \varepsilon_{n+1}^{p}=\varepsilon_{n}^{p}+\Delta \gamma, \quad \mathbb{C}=2 G\left[1-\frac{3 \Delta \gamma G}{q_{n+1}^{\text {trial }}}\right] \mathbb{I}_{d}+6 G^{2}\left[\frac{\Delta \gamma}{q_{n+1}^{\text {rial }}}-\frac{\Delta t}{\eta+\Delta t\left(3 G+f_{n+1}^{s} H\right)}\right] \overline{\mathbf{N}}_{n+1} \otimes \overline{\mathbf{N}}_{n+1}+K \mathbf{I} \otimes \mathbf{I}, \\
& \left(\mathbb{I}_{d}\right)_{i j k l}=\frac{1}{2}\left[\delta_{i k} \delta_{j l}+\delta_{i l} \delta_{j k}\right]-\frac{1}{3} \delta_{i j} \delta_{k l}, \quad \overline{\mathbf{N}}_{n+1}=\frac{\mathbf{s}_{n+1}}{\left\|\mathbf{s}_{n+1}\right\|}, \quad H=\left.\frac{\mathrm{d} \sigma_{y}}{\mathrm{~d} \varepsilon^{p}}\right|_{\varepsilon_{n+1}^{p}} .
\end{aligned}
$$

\section{Model for precipitation hardening}

\subsection{Evolution equations of the KWN model}

Artificial ageing after homogenization of precipitation hardened aluminium alloys produces a high density of fine hardening precipitates in the aluminium matrix, which efficiently hinder the movement of dislocations. The high temperatures in the fusion zone and the HAZ of welded aluminium structures lead to dissolution and growth of the precipitates and a significant decrease in the yield strength.

In principle, solid-state precipitation is described by nucleation, growth and coarsening (Wagner et al., 2001). By means of classic nucleation theory, it is possible to calculate the size and the amount of spherical particles that are nucleating at a specific time by the critical radius $r^{*}$ and the nucleation rate $j$. The critical radius $r^{*}$ describes the radius where neither growth nor dissolution occurs. The nucleation process produces new precipitates that are slightly larger than the critical radius $r^{*}$. After nucleation, precipitates grow by absorbing atoms from the supersaturated matrix.

The KWN model allows the description of nucleation, growth and coarsening for solid state precipitates. The majority of investigations using the KWN model assume a spherical particle shape. Nevertheless, few approaches investigate possibilities to generalize the KWN model to non-spherical particles shapes (Holmedal et al., 2016; Du et al., 2016). The KWN model for spherical precipitates describe the evolution of precipitates via a particle size distribution that is a continuous function of time $t$ and radius $r$. Every particle in the control volume is counted by a size distribution function $N(r, t)$. Depending on the critical radius, the particles grow or dissolve with the velocity/growth rate $v$. By 
knowing the growth and the nucleation rate, the development of the particle size distribution is described according to Myhr and Grong (2000) by

$$
\frac{\partial N}{\partial t}=-\frac{\partial}{\partial r}[N v]+j \delta\left(r-r^{*}\right)
$$

Here denotes $\delta(\bullet)$ the delta distribution function. In general, the precipitation sequence for aluminium-magnesiumsilicon alloys shows a complex precipitation sequence of different coherent, semi-coherent and incoherent precipitates. In the applied model by Myhr et al. (2004) for Al-Mg-Si alloys, a single precipitation class for $\beta^{\prime \prime}$-phase with stoichiometric chemical composition $\mathrm{Mg}_{5} \mathrm{Si}_{3}$ is considered.

During growth, the constituent elements of the precipitates are absorbed from the supersaturated aluminium matrix, which results in an increase of the volume fraction of the precipitating phase. The supersaturation of the constituent elements in the matrix decreases and the system moves closer to a state of thermodynamic equilibrium. The depletion of the matrix by constituent elements of the phase is considered by a mass balance. The mass balance for spherical shaped particles with volume fraction $f_{p}$ gives

$$
C_{i}^{\alpha}=\frac{C_{i}^{0}-f_{p} C_{i}^{\beta}}{1-f_{p}} \quad i=\{\mathrm{Mg}, \mathrm{Si}\} \quad \text { with } \quad f_{p}=\sum_{k} \frac{4}{3} \pi r_{k}^{3} N_{k}
$$

The solution for this equation is obtained by utilizing standard solution methods of numerical fluid dynamics. In particular, a finite volume method in combination with a linear upwind scheme, as outlined by Myhr and Grong (2000), has been used. The nucleation rate $j$, which describes how many particles nucleate at a distinct time at a given temperature $T$, is modelled via simplified classic nucleation theory for binary alloys under assumption of a dilute solution, see also Myhr and Grong (2000):

$$
j=j_{0} \exp \left(\left[-\frac{A_{0}}{R T}\right]^{3}\left[\frac{1}{\ln \left(C_{M g}^{\alpha} / C_{M g, e}^{\alpha}\right)}\right]^{2}\right) \exp \left(-\frac{Q_{d}}{R T}\right),
$$

where $j_{0}$ is a pre-exponential coefficient, $Q_{d}$ represents the activation energy for diffusion, $R$ denotes the gas constant, $C_{M g, e}^{\alpha}$ is the equilibrium concentration of magnesium in the matrix in weight percent and $A_{0}$ is a constant with same dimension as the activation energy for nucleation. Nonetheless, because of the rapid heating and cooling rates, the material is far from thermodynamic equilibrium. Because of significant higher vacancy site fractions, the diffusion constants can differ significantly from the values at equilibrium. In general, nucleation and growth is highly dependent on the thermodynamic input. It is well established that nucleation at high elevated temperatures leads to nucleation and growth of more stable phases, which show only minor influence on the yield stress (Edwards et al., 1998). The model of Myhr and co-workers (Myhr and Grong, 2000; Myhr et al., 2001, 2004) shows remarkable nucleation during cooling in a temperature range between $300^{\circ} \mathrm{C}$ and $400^{\circ} \mathrm{C}$ for the investigated temperature field with parameters from Table 2. Recent thermodynamic descriptions by Povoden-Karadeniz et al. (2013) show that the temperature range where precipitations of $\beta^{\prime \prime}$ are stable, is significantly smaller. Therefore, it has been assumed in this contribution that nucleation during the cooling of the welding process for hardening precipitates is negligible and therefore disregarded. In the KWN model, growth is described by a binary diffusion process, assuming that only the diffusion of magnesium has an influence on the precipitation process. The calculation of the interface concentration of the matrix $C_{M g}^{\alpha}$ at the matrix-precipitate-interface has to consider the Gibbs-Thomson effect (Perez, 2005). Here, the interface concentration of the matrix is calculated following Myhr and Grong (2000) by the equilibrium concentration of magnesium in the matrix $C_{M g}^{\alpha, e}$, the interfacial energy $\sigma$ and the molar volume of the precipitating phase $V_{m}^{p}$ by the approximation

$$
C_{M g}^{\alpha, i}=C_{M g, e}^{\alpha} \exp \left(\frac{2 \sigma V_{m}}{r R t}\right) .
$$

The equilibrium concentration is given by an exponential fitting, according to Myhr et al. (2004) as

$$
C_{M g, e}^{\alpha}=290 \exp \left(-\frac{41000}{R T}\right) \text { in [wt-\%]. }
$$


The particle is going to grow, if the interface concentration of the precipitate $C_{M g}^{\alpha, i}$ is exceeded by the concentration of magnesium in the aluminium matrix $C_{M g}^{\alpha}$. For a stoichiometric particle with radius $r$ and magnesium concentration $C_{M g}^{\beta}$, the growth rate $v$ and critical radius are calculated by

$$
v=\frac{C_{M g}^{\alpha}-C_{M g}^{\alpha, i}}{C_{M g}^{\beta}-C_{M g}^{\alpha, i}} \frac{D}{r} \quad \text { and } \quad r^{*}=\frac{2 \sigma V_{m}}{R T}\left[\ln \left(\frac{C_{M g}^{\alpha}}{C_{M g, e}^{\alpha}}\right)\right]^{-1} .
$$

\subsection{Yield strength and work hardening model}

The high strength of precipitation hardened aluminium alloys results primarily from the precipitates. A combined yield strength and work hardening model has been established by Myhr et al. (2001, 2004, 2010). The model is based on the Fleischer-Friedel-Point-Obstacle approximation which is described by Ardell (1985) and Reppich (1993). The yield stress $\sigma_{y}$, representing one key parameter in the material model, see Section 4 and Eq.(4.22), is additively composed of contributions from intrinsic yield strength $\sigma_{i}$, solute solution strengthening $\sigma_{s s}$, particle strengthening $\sigma_{p}$ and a term which takes into account the effect of dislocation strengthening $\sigma_{d}$

$$
\sigma_{y}=\sigma_{i}+\sigma_{s s}+\sigma_{p}+\sigma_{d}
$$

The particle strengthening $\sigma_{p}$ is calculated via the mean obstacle strength $\bar{F}$, Taylor factor $M$, Burgers vector $b$ and the effective particle spacing $l$. The effective particle spacing depends on the applied stresses in the glide plane. According to Reppich (1993), large obstacle strength leads to larger bending of the dislocation line; therefore, the probability increases to come in contact with more obstacles. This effect is taken into account via the Friedl formalism, following Myhr et al. (2004) leads to

$$
\sigma_{p}=\frac{M \bar{F}}{b l}=\frac{M}{b^{2} \sqrt{G}} \sqrt{\frac{N_{v} \bar{r}}{\beta}} \bar{F}^{3 / 2} .
$$

The constant describing dislocation line tension is $\beta$ and $\bar{r}$ denotes the mean radius. The number of particles per unit volume is $N_{v}$. The average obstacle strength $\bar{F}$ takes into account the different mechanisms of how a dislocation can interact with a precipitate. As shown by Myhr et al. (2001), the mean obstacle strength is calculated by averaging over the contributions from the different size classes

$$
\bar{F}=\frac{\sum_{i} N_{i} F_{i}}{\sum_{i} N_{i}}
$$

Depending on the radius of the size class, the precipitates are sheared or bypassed by the dislocations. The precipitations that are smaller than the critical radius $r_{c}$ are sheared and their contribution to the mean obstacle strength, see also Myhr et al. (2001), is calculated via

$$
F_{i}=2 \beta G b^{2}\left[\frac{r_{i}}{r_{c}}\right] .
$$

In contrast, the mean obstacle strength of a single size class for a strong particle larger than the critical radius $r_{c}$ is determined by

$$
F_{i}=2 \beta G b^{2} .
$$

As suggested by Myhr et al. (2001), the effect of solid solution is calculated as

$$
\sigma_{s S}=k_{M g}\left[C_{M g}^{\alpha}\right]^{2 / 3}+k_{S i}\left[C_{S i}^{\alpha, e f f}\right]^{2 / 3}+k_{C u}\left[C_{C u}^{\alpha}\right]^{2 / 3} \quad \text { with } \quad C_{S i}^{\alpha, e f f}=C_{S i}^{\alpha}-0.33\left[C_{0, F e}^{\alpha}+C_{0, M n}^{\alpha}\right] .
$$

The equation for work hardening is given by the Taylor-equation

$$
\sigma_{d}=\alpha M G b \sqrt{\rho_{s}+\rho_{g}}
$$

where $\alpha$ is a adjustable parameter. Further, the dislocations are separated in statistically stored dislocations $\rho_{s}$ and geometrically necessary dislocations $\rho_{g}$. The Kocks-Mecking equation is used for describing the evolution of statistically stored dislocations

$$
\frac{d \rho_{s}}{d \varepsilon_{p}}=k_{1} \sqrt{\rho_{s}}-k_{2} \rho_{s}
$$


According to this equation, the first term $k_{1}$ describes the generation and $k_{2}$ the dynamic recovery of dislocations. The integration of this equation yields

$$
\rho_{s}=\left[\frac{k_{1}}{k_{2}}\right]^{2}\left[1-\exp \left(\frac{-k_{2} \varepsilon_{p}}{2}\right)\right]^{2} .
$$

The dynamic recovery is influenced by the mean solid content, described by

$$
k_{2}=k_{1} \frac{\alpha M G b}{k_{3}\left[C_{M g}^{\alpha}+0.5 C_{S i}^{\alpha, e f f}\right]^{3 / 4}} .
$$

The contribution from non shareable particles is derived by using a normalizing procedure introducing a reference alloy with volume fraction of Orowan precipitations $f_{0, O}^{r e f}$. The geometrically necessary dislocation density increases up to a certain limit. After reaching this limit, recovery mechanisms are dominant. The critical plastic strain $\varepsilon_{c}$ which denotes this value is

$$
\varepsilon_{c}=\left[\frac{f_{0, O}^{r e f}}{f_{0, O}}\right] \varepsilon_{c}^{r e f} .
$$

As outlined, particles that are larger than the the critical radius contribute to the Orowan mechanism; therefore,

$$
f_{0, O}=\sum_{i} \frac{4}{3} \pi r_{i}^{3} N_{i} \quad \text { and } \quad \lambda_{g, 0}=\left[8 \sum_{i} r_{i}^{2} N_{i}^{2}\right]^{-1} \text { if } \quad r_{i}>r_{c} .
$$

The geometrically stored dislocations $\rho_{g}$ are then determined by

$$
\rho_{g}=\rho_{g, s}^{r e f} \frac{\lambda_{g, 0}^{r e f} \varepsilon_{p}}{\lambda_{g, 0} \varepsilon_{c}^{r e f}} \quad \text { when } \quad \varepsilon_{p}<\varepsilon_{c} \quad \text { and } \quad \rho_{g}=\rho_{g, s}^{r e f} \frac{\lambda_{g, 0}^{r e f} \varepsilon_{c}}{\lambda_{g, 0} \varepsilon_{c}^{r e f}} \quad \text { when } \quad \varepsilon_{p} \geq \varepsilon_{c} .
$$

For the numerical procedure, the definitions given in Eq.(5.45) and Eq.(5.41) are embedded into the Taylor Eq.(5.39). The required parameters for the KWN model as well as for the work hardening and yield strength model are summarized in Table 3. These parameters are taken from previous studies of Myhr et al. (2001, 2004, 2010), where the calibration is presented in detail.

\section{Experimental and numerical results}

\subsection{Calibration of the temperature field model}

The identification of the parameters for the temperature field model had been performed under the assumption of adiabatic boundary conditions. The implemented algorithm allows a fast identification process of the parameters due to the high efficient formulation. Additionally, the numerical error can be controlled easily by the used adaptive integration procedure. A crucial point regarding the identification of the parameters for the temperature field model is the utilized information. In general, measurement devices for welding offer only a limited spacial resolution accompanied with an inherent time delay as mentioned in Slania et al. (2007). There the problems occurring in measuring temperatures for the welding of steel are discussed. The temperature measurements for welding of aluminium suffer similar problems. However, it has to be considered that aluminium alloys have significant higher thermal conductivities.

The temperatures curves of the measurement as well as for the simulation for the selected points are shown in Fig.5(a). The parameters resulting from the inverse temperature identification procedure are given in Table 2. The resulting temperature field for the $\mathrm{x}-\mathrm{y}$ plane is shown in Fig.5(b) at time $\mathrm{t}=1.5$ seconds. It is worth noting, that the width of the weld seam generally reaches its maximum behind the centre of the heat source. Form inspection of Fig.5(b) it is noticed that the model shows a slight overestimation of the weld seam width if we assuming a liquidus temperature of $640^{\circ} \mathrm{C}$ according to Mills (2002) for AA6061.

A problem that is often encountered in temperature measurements of laser beam welding processes is the occurrence of unsatisfying scatter for measurements next to the fusion zone. The investigated solution of the heat transfer equation gives an impressive explanation for the occurrence of this measurement problem. From inspection of Fig.5 large changes in temperatures occur at very small distances. 
Table 3: Parameters of the KWN model and the yield strength and work hardening model

\begin{tabular}{|l|l|l|l|l|}
\hline Parameter & Symbol & Value & Unit & Reference \\
\hline Magnesium content in $\mathrm{Mg}_{5} \mathrm{Si}_{3}$ & $C_{M g}^{\beta}$ & 59 & $\mathrm{wt}-\%$ & Myhr et al. (2001) \\
Diffusion coefficient & $D_{0}$ & $2.2 \cdot 10^{-4}$ & $\mathrm{~m} / \mathrm{s}$ & Myhr et al. (2004) \\
Activation energy for diffusion & $Q_{d}$ & 130 & $\mathrm{~kJ} / \mathrm{mol}$ & Myhr et al. (2004) \\
Nucleation barrier & $A_{0}$ & 18 & $\mathrm{~kJ} / \mathrm{mol}$ & Myhr et al. (2004) \\
Nucleation rate & $j$ & $3.07 \cdot 10^{36}$ & $\mathrm{~kJ} / \mathrm{mol}$ & Myhr et al. (2004) \\
Molar volume of precipitate & $V_{m}^{p}$ & $7.62 \cdot 10^{-5}$ & $\mathrm{~m}^{3} / \mathrm{mol}$ & Myhr et al. (2004) \\
Interfacial energy & $\sigma$ & 0.26 & $\mathrm{~J} / \mathrm{m}^{2}$ & Myhr et al. (2004) \\
\hline Critical radius & $r_{c}$ & 5 & $\mathrm{~nm}$ & Myhr et al. (2001) \\
Taylor factor & $M$ & 3.1 & & Myhr et al. (2001) \\
Constant dislocation line tension & $\beta$ & 0.36 & & Myhr et al. (2001) \\
Shear modulus & $G$ & $2.7 \cdot 10^{10}$ & $\mathrm{~N} / \mathrm{m}^{2}$ & Myhr et al. (2001) \\
Magnitude of burgers vector & $b$ & $2.84 \cdot 10^{-10}$ & $\mathrm{~m}$ & Myhr et al. (2001) \\
Intrinsic stress & $\sigma_{i}$ & 10 & $\mathrm{MPa}$ & Myhr et al. (2001) \\
Solid solution parameter for Mg & $k_{M g}$ & 66.3 & $\mathrm{MPa}$ & Myhr et al. (2001) \\
Solid solution parameter for Si & $k_{S i}$ & 29.0 & $\mathrm{MPa}$ & Myhr et al. (2001) \\
\hline Parameter of Taylor-Eq. & $\alpha$ & 0.3 & & Myhr et al. (2010) \\
First parameter of KM-Eq. & $k_{1}$ & $4 \cdot 10^{8}$ & $1 / \mathrm{m}$ & Myhr et al. (2010) \\
Second parameter of KM-Eq. & $k_{3}$ & $2 \cdot 10^{8}$ & $\left(N w t-\% \%^{3 / 4}\right) / \mathrm{m}^{2}$ & Myhr et al. (2010) \\
Geometric slip distance of reference & $\lambda_{g, 0}^{r e f}$ & $4.1 \cdot 10^{-7}$ & $\mathrm{~m}$ & Myhr et al. (2010) \\
Reference volume fraction of non sheareable particels & $f_{0}^{r e f}$ & 0.0109 & $\mathrm{~m}$ & Myhr et al. (2010) \\
Saturation value for $\rho_{g}$ for reference alloy & $\rho_{g, s}^{r e f}$ & $4.93 \cdot 10^{13}$ & $1 / \mathrm{m}^{2}$ & Myhr et al. (2010) \\
Threshold strain & $\varepsilon_{c}^{r e f}$ & 0.05 & & Myhr et al. (2010) \\
\hline
\end{tabular}




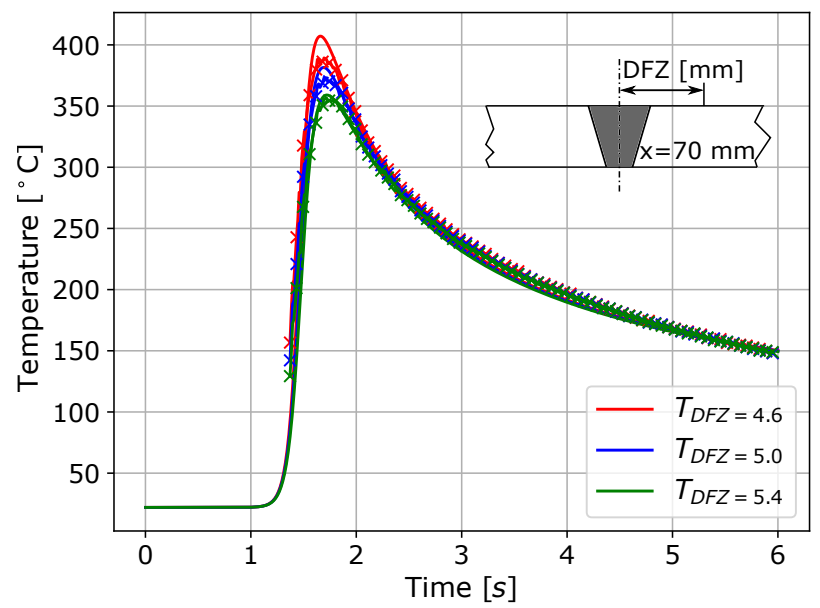

(a)

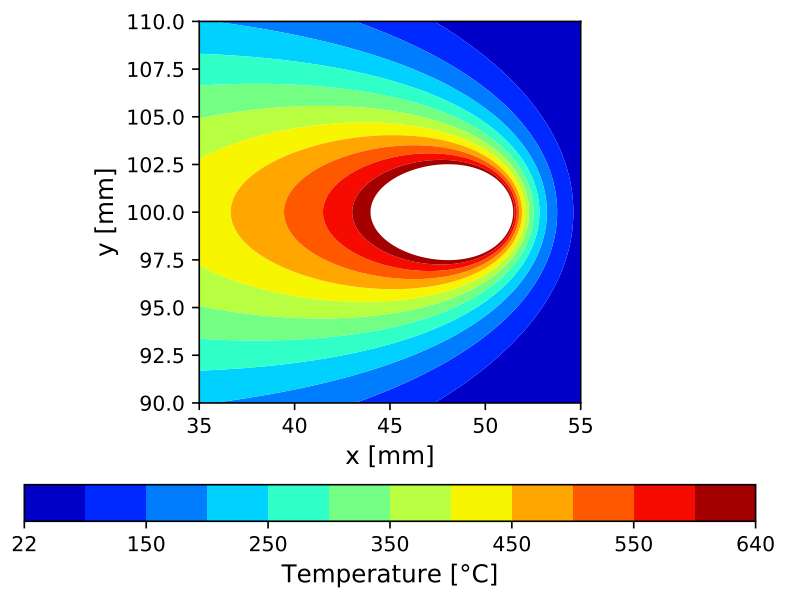

(b)

Figure 5: Results of the model of the temperature field based on identified parameters summarized in Table 2. (a) Measured temperature (crossed lines) compared to corresponding simulated temperatures (solid lines) with respect to distance of the center of the fusion zone (DFZ) . (b) Section from temperature field in $\mathrm{x}-\mathrm{y}$ plane for $\mathrm{z}=0 \mathrm{~mm}$. Material above the melting temperature $\left(640^{\circ} \mathrm{C}\right)$ is identified by white colour, indicating the width of the fusion zone.

\subsection{Comparison of mechanical properties}

The investigated material is welded in T6 condition. The initial particle size distribution for the T6 temper state, used as initial condition for the KWN model, is calculated via 15000 seconds ageing from solid solution by using the nucleation and growth theory as outlined in Section 5.1. The yield stress immediately after welding is calculated by coupling the temperature field model to the KWN model under the assumption that nucleation is not occurring during cooling of the weld. This assumption is made, because the model shows a pronounced nucleation during cooling in the temperature range between $300^{\circ} \mathrm{C}$ and $400^{\circ} \mathrm{C}$.

The microhardness profile, measured transverse to the weld seam, is presented in Fig.6(a). The hardness decreases significantly in the fusion and the HAZ. A common assumption for precipitation hardened aluminium alloys is to approximate the yield strength or the hardness of the material by a linear relationship (Myhr et al., 2001; Bardel et al., 2016a). Therefore, the decrease is related to a reduction in yield strength.

From the hardness measurement it is observed that the fusion zone shows the lowest hardness values of the weld seam after welding. The hardness of the HAZ is extensively characterized by a plateau. The transition between the HAZ and the base material is characterized by a strong increase in hardness.

Simulation results for the yield strength are shown in Fig.6(a) as well. Additionally, the spatial distribution of the particle volume fraction and yield strength determined from the precipitation hardening model are shown in Fig.6(b), for a two dimensional cross section. In the fusion zone and the HAZ the rise of temperature leads to a increase of the critical radius in the KWN model. Precipitates smaller than the critical radius are unstable, which results in a severe decrease of the particle number density and the volume fraction of precipitates, respectively.

The temporal response of the point $\mathbf{x}=(50 \mathrm{~mm}, 94 \mathrm{~mm}, 0 \mathrm{~mm})$, which belongs to the HAZ is investigated in Fig.7. The heating-cooling-curve and the corresponding yield strength are illustrated in Fig.7(a). Additionally, the volume fraction and yield strength are shown in Fig.7(b). The figures show that large amounts of precipitates in the HAZ become unstable and dissolve during the welding process.

The inspection of Fig.6(a) shows that the microstructure model delivers a slightly smaller HAZ than expected from the microhardness measurements. The unambiguous identification of the reason for this difference between simulation and experiment is difficult because deviations of the temperature field model as well as the KWN model are directly linked. An increase of the welding power $Q$ results in a larger fusion zone and HAZ. Nevertheless, the modelling error resulting from the simplified thermodynamic input and the inherent assumptions of the KWN method might also provoke these differences. For example, the consolidation of different phases via one particle size distribution might overestimate the stability of some phases. This might be the reason for the very sharp drop shown in the simulation 


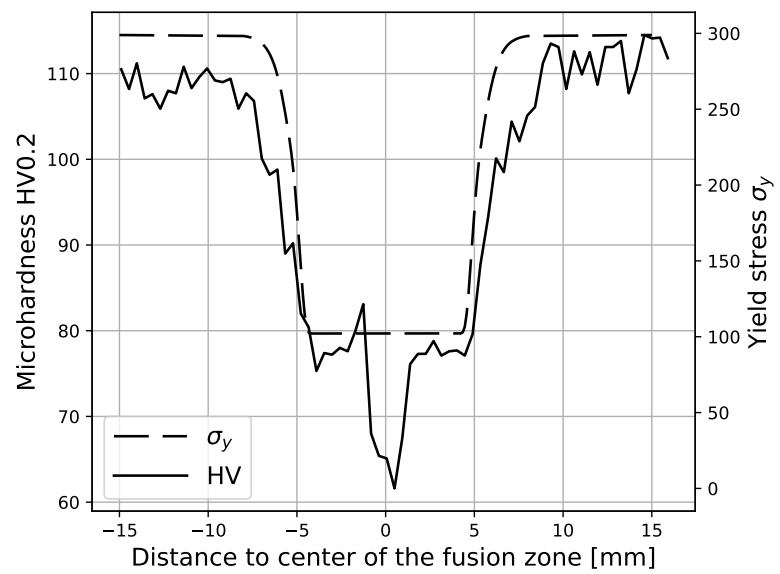

(a)

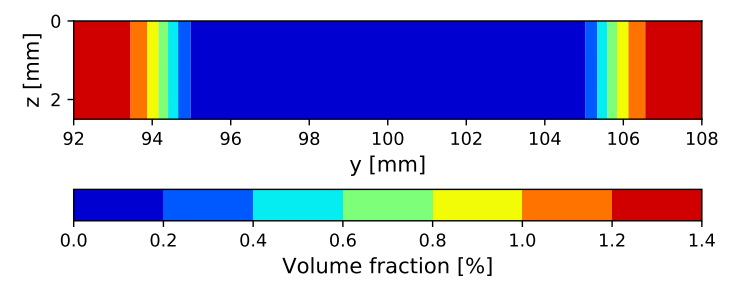

(b)

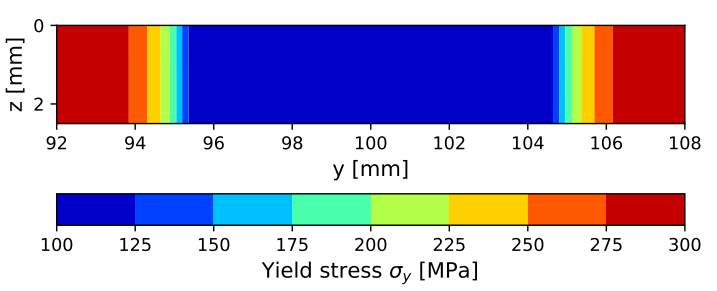

(c)

Figure 6: (a) Simulated yield stress (dashed line) after welding and measured microhardness (solid line) for AA6082-T6. Obtained volume fraction (b) and yield strength distribution (c) after welding.

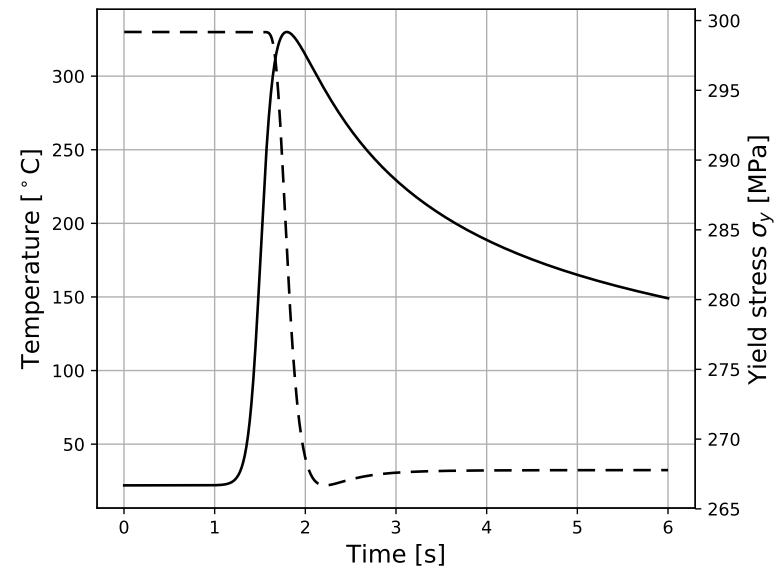

(a)

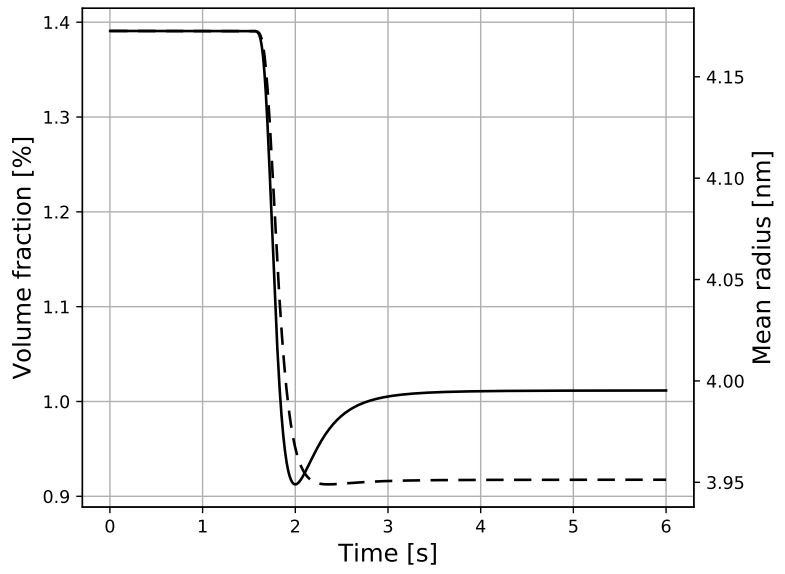

(b)

Figure 7: (a): Temperature evolution for the point $\mathbf{x}=(50 \mathrm{~mm}, 94 \mathrm{~mm}, 0 \mathrm{~mm})$ in the HAZ (solid line). Evolution of the yield stress during welding (dashed line). (b): Evolution of volume fraction (dashed line) and mean radius (solid line) for corresponding temperature curve as shown in (a). 


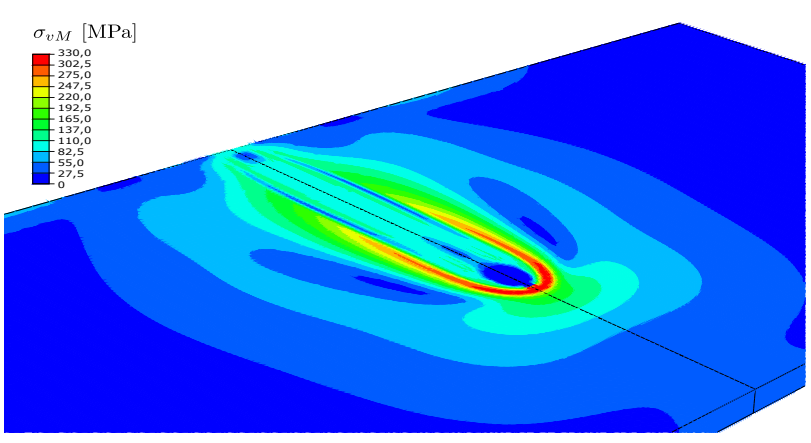

(a)

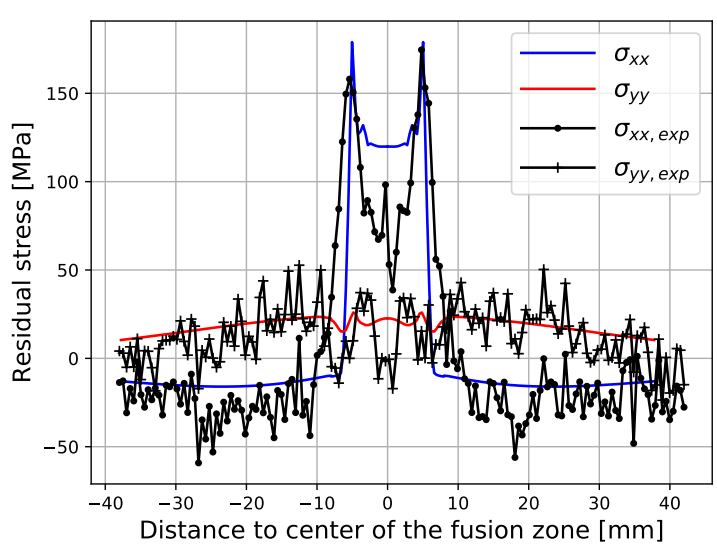

(b)

Figure 8: (a) Von Mises yield stress during the welding process. (b) Comparison of measured to simulated residual stresses: Measured residual stresses in longitudinal direction $\sigma_{x x \text {, exp }}$; Measured residual stresses for transversal direction $\sigma_{y y, \text { exp }}$. Simulation results are shown in coloured lines.

results for the yield strength, see Fig.6(a). Nonetheless, the start of the plateau as well as the magnitude of the width of the HAZ are well predicted by the model.

A very interesting effect is the observed drop of the microhardness in the fusion zone. By inspection of the etched micrograph in Fig.2(b), the morphology of the fusion zone shows a considerably different morphology compared to the HAZ. According to the contribution of Pakdil et al. (2011) this morphology is attributable to segregation. In a weld seam made of AA6061, Bardel et al. (2016b) found the equilibrium $\beta$-phase as a result from solidification. The constituent elements of non hardening phases are absorbed from the aluminium matrix and can not contribute to solid solution hardening or even to the formation of new precipitates and, as a result, lead to a decrease in yield strength. Obviously, this effect is not addressed by the investigated KWN model; therefore, the drop in the fusion zone cannot be represented by the model.

\subsection{Residual stresses}

Plasticity is only occurring in the fusion zone and in the HAZ. Hence, it is sufficient to define the material model as well as the KWN model only for elements in the fusion zone and the HAZ to decrease the computational effort. The estimation of the width of the HAZ can be done without doing a previous finite element simulation.

Residual stresses are a result of the inhomogeneous temperature distribution characteristic of welding processes. According to the schematic description given by Radaj (2003) a zone of compressional stresses located on a semicircle around the front of the fusion zone is present during welding. After reaching the locally distributed maximum temperature, the plasticised material begins to change from a state of compression stresses to a state of tensile stresses. This qualitative descriptions is in accordance with the results of the multiscale finite element simulations performed. Fig.8(a) shows the von Mises yield stress during the welding process. The von Mises stress virtually vanishes for liquid material as expected from the material model.

The results of the finite element simulations affirm the hypothesis of plane stress conditions. The results of the finite element simulations virtually show no residual stresses in thickness direction. Additionally, the shear stresses occurring in the simulation are negligible. The results for a line scan performed for constant $x=50 \mathrm{~mm}$, corresponding to the middle of the specimen, is shown in Fig.8(b). It shows residual stresses for the longitudinal as well as transversal directions. In general, the residual stresses for the $\sigma_{x x}$-component are significantly less pronounced than the residual stresses for the $\sigma_{y y}$-component.

The 2D-maps of measured residual stresses for the longitudinal direction ( $\sigma_{x x}$-component) are illustrated in Fig.9(a). The associated transverse direction is shown in Fig.9(b). The residual stresses are presented in y-direction, relative to 

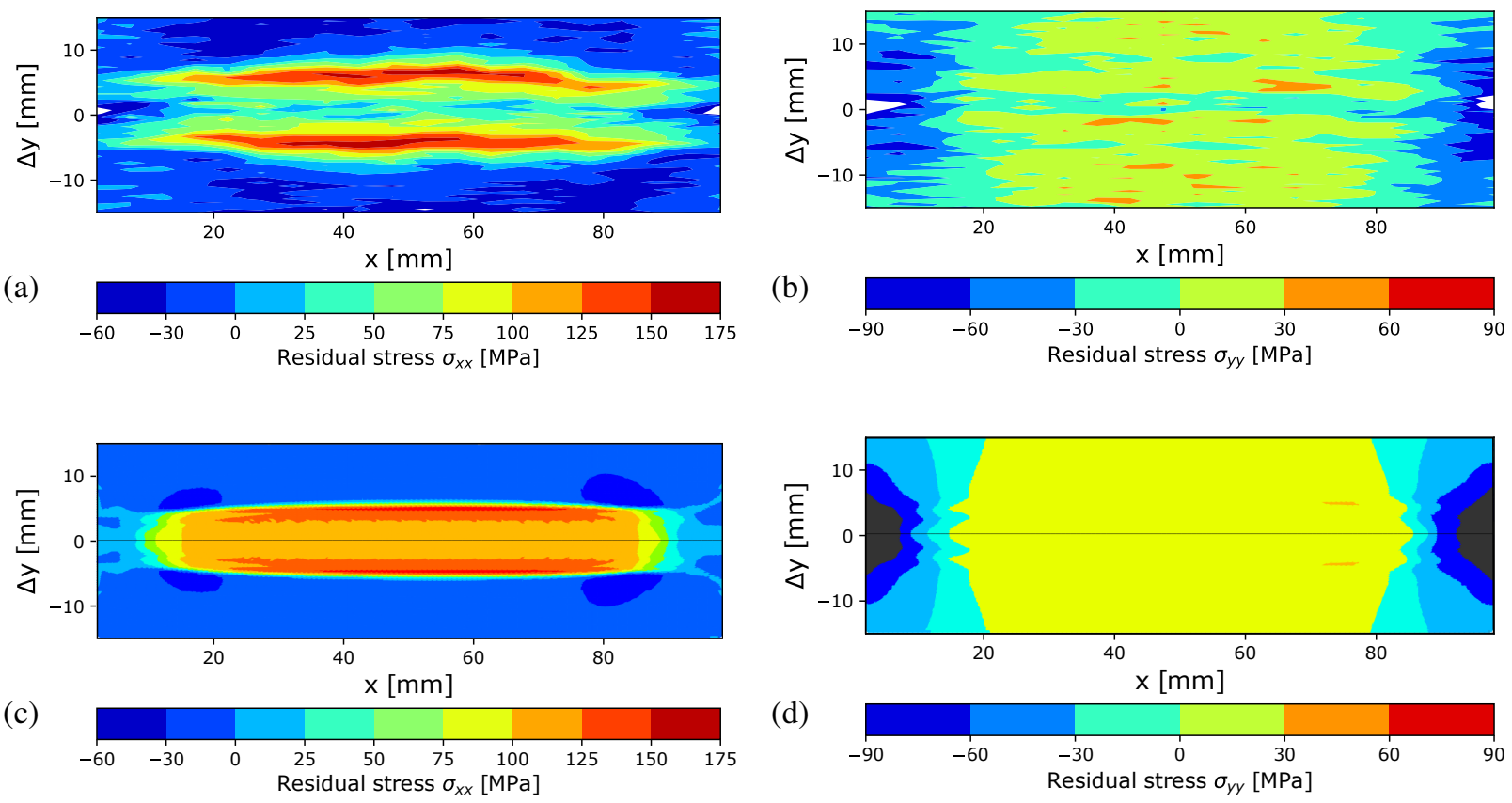

Figure 9: Residual stresses after welding for AA6082 measured under the assumption of plane stress by synchrotron X-ray diffraction: (a) Residual stresses for the $\sigma_{x x}$-component. (b) Residual stresses for the $\sigma_{y y}$-component. Residual stresses after welding obtained via finite element method: (c) Residual stresses of the $\sigma_{x x}$-component. (d) Residual stresses of the $\sigma_{y y}$-component.

the centre of the fusion zone. The largest tensile residual stresses in the simulation as well as in the experiment occur in the HAZ. This corresponds to results given by Staron et al. (2009). An area corresponding to the measured residual stress field is used for extracting the residual stresses from the finite element simulation for reasons of comparison. The residual stress fields from the finite element simulations are shown in Fig.9(c) for the $\sigma_{x x}$-component and for the $\sigma_{y y}$-component in Fig.9(d). Simulation and experiment are in agreement, showing that the highest residual stresses exist in the HAZ. Also, the maximum level of residual stresses is calculated with a satisfying degree of accuracy. Nonetheless, the residual stresses in the fusion zone are overestimated because the yield strength of the fusion zone is higher than expected from experiments, as outlined in the previous subsection.

\section{Conclusion}

A multiscale numerical model for describing laser beam welding of precipitation hardened aluminium alloys has been presented and successfully applied to welded AA6082-T6 sheets. These sheets were investigated experimentally by micrographs, temperature and microhardness measurements and synchrotron X-ray-diffraction. A solution of the heat equation for a cuboid domain has been presented and successfully applied for calibration of the temperature field. The temperature field has been used for simulation of the precipitation kinetics via a KWN model. A material model which considers phenomenologically the change from fluid phase to solid phase has been coupled to the KWN model and used in finite element software ABAQUS for calculation of residual stresses. Good agreement to the experimental results is obtained for temperature, hardness and residual stress distributions in the simulation. The used hypotheses of plane stress conditions, used for the evaluation of the synchrotron-X-ray-diffraction images, is confirmed by evaluating the residual stress field in the simulation. Thanks to the integration of the microstructural model, the typical ' $M$ ' profile of the longitudinal residual stresses was successfully reproduced by the investigated model. 


\section{Acknowledgment}

We acknowledge Deutsches Elektronen-Synchroton (DESY), Germany, for using synchrotron radiation at beamline P07B of Helmholtz-Zentrum Geesthacht. The authors would like to thank Dr. Josephin Enz for their valuable support using the laser laboratory at Helmholtz-Zentrum Geesthacht and Falk Dorn for the support during metallographic preparation. Furthermore, we like to thank Dr. Ingo Scheider for his support on the simulation environment.

\section{References}

Ardell, A. J., Dec 1985. Precipitation hardening. Metallurgical Transactions A 16 (12), 2131-2165.

Bardel, D., Fontaine, M., Chaise, T., Perez, M., Nelias, D., Bourlier, F., Garnier, J., 2016a. Integrated modelling of a 6061-T6 weld joint: From microstructure to mechanical properties. Acta Materialia 117, $81-90$.

Bardel, D., Nelias, D., Robin, V., Pirling, T., Boulnat, X., Perez, M., 2016b. Residual stresses induced by electron beam welding in a 6061 aluminium alloy. Journal of Materials Processing Technology 235, $1-12$.

Bardel, D., Perez, M., Nelias, D., Deschamps, A., Hutchinson, C., Maisonnette, D., Chaise, T., Garnier, J., Bourlier, F., 2014. Coupled precipitation and yield strength modelling for non-isothermal treatments of a 6061 aluminium alloy. Acta Materialia 62, 129-140.

Beck, J. V., Cole, K. D., Haji-Sheikh, A., Litkouhi, B., 1992. Heat conduction using Green's functions. Hemisphere Publishing Corporation London.

Cervera, M., Agelet De Saracibar, C., Chiumenti, M., 1999. Thermo-mechanical analysis of industrial solidification processes. International Journal for Numerical Methods in Engineering 46 (9), 1575-1591.

Chiumenti, M., Cervera, M., Salmi, A., De Saracibar, C. A., Dialami, N., Matsui, K., 2010. Finite element modeling of multi-pass welding and shaped metal deposition processes. Computer Methods in Applied Mechanics and Engineering 199 (37), 2343-2359.

Cline, H., Anthony, T., 1977. Heat treating and melting material with a scanning laser or electron beam. Journal of Applied Physics 48 (9), 3895-3900.

Dal, M., Fabbro, R., 2016. An overview of the state of art in laser welding simulation. Optics \& Laser Technology 78, 2-14.

Du, Q., Holmedal, B., Friis, J., Marioara, C. D., 2016. Precipitation of non-spherical particles in aluminum alloys Part ii: numerical simulation and experimental characterization during aging treatment of an Al-Mg-Si alloy. Metallurgical and Materials Transactions A 47 (1), 589-599.

Du, Q., Tang, K., Marioara, C. D., Andersen, S. J., Holmedal, B., Holmestad, R., 2017. Modeling over-ageing in Al-Mg-Si alloys by a multi-phase CALPHAD-coupled kampmann-wagner numerical model. Acta Materialia 122, 178-186.

Edwards, G., Stiller, K., Dunlop, G., Couper, M., 1998. The precipitation sequence in Al-Mg-Si alloys. Acta Materialia 46 (11), 3893 - 3904

Esmaeili, S., Wang, X., Lloyd, D., Poole, W., 2003. On the precipitation-hardening behavior of the Al-Mg-Si-Cu alloy aa6111. Metallurgical and Materials Transactions A 34 (13), 751-763.

Fitzpatrick, M. E., Lodini, A., 2003. Analysis of residual stress by diffraction using neutron and synchrotron radiation. CRC Press.

Goldak, J., Chakravarti, A., Bibby, M., 1984. A new finite element model for welding heat sources. Metallurgical and Materials Transactions B 15 (2), 299-305.

Hammersley, A., Svensson, S., Hanfland, M., Fitch, A., Hausermann, D., 1996. Two-dimensional detector software: from real detector to idealised image or two-theta scan. International Journal of High Pressure Research 14 (4-6), 235-248.

Hammersley, A., et al., 1997. FIT2D: an introduction and overview. European Synchrotron Radiation Facility Internal Report ESRF97HA02T 68, 58.

Herrnring, J., Klusemann, B., 2017. Finite element modeling of laser beam welding for residual stress calculation. PAMM 17 (1), 415-416.

Holmedal, B., Osmundsen, E., Du, Q., 2016. Precipitation of non-spherical particles in aluminum alloys Part i: Generalization of the KampmannWagner Numerical Model. Metallurgical and Materials Transactions A 47 (1), 581-588.

Karkhin, V., Pittner, A., Schwenk, C., Rethmeier, M., 2011. Simulation of inverse heat conduction problems in fusion welding with extended analytical heat source models. Frontiers of Materials Science 5 (2), 119.

Lindgren, L.-E., 2001. Finite element modeling and simulation of welding Part 1: increased complexity. Journal of thermal stresses 24 (2), 141-192.

Marioara, C., Andersen, S., Jansen, J., Zandbergen, H., 2001. Atomic model for GP-zones in a 6082 Al-Mg-Si system. Acta Materialia 49 (2), 321 -328 .

Mills, K. C., 2002. Recommended values of thermophysical properties for selected commercial alloys. Woodhead Publishing.

Moraitis, G., Labeas, G., 2008. Residual stress and distortion calculation of laser beam welding for aluminum lap joints. Journal of materials processing technology $198(1), 260-269$.

Myhr, O., Grong, Ø., 2000. Modelling of non-isothermal transformations in alloys containing a particle distribution. Acta Materialia 48 (7), $1605-$ 1615 .

Myhr, O., Grong, Ø., Andersen, S., 2001. Modelling of the age hardening behaviour of Al-Mg-Si alloys. Acta Materialia 49 (1), 65-75.

Myhr, O., Grong, Ø., Fjær, H., Marioara, C., 2004. Modelling of the microstructure and strength evolution in Al-Mg-Si alloys during multistage thermal processing. Acta Materialia 52 (17), 4997-5008.

Myhr, O. R., Grong, Ø., Pedersen, K. O., 2010. A combined precipitation, yield strength, and work hardening model for Al-Mg-Si alloys. Metallurgical and Materials Transactions A 41 (9), 2276-2289.

Nélias, D., Jullien, J.-F., Deloison, D., et al., 2010. Experimental investigation and finite element simulation of laser beam welding induced residual stresses and distortions in thin sheets of AA6056-T4. Materials Science and Engineering: A 527 (12), 3025-3039.

Pakdil, M., am, G., Kocak, M., Erim, S., 2011. Microstructural and mechanical characterization of laser beam welded AA6056 Al-alloy. Materials Science and Engineering: A $528(24), 7350-7356$

Perez, M., 2005. Gibbs-Thomson effects in phase transformations. Scripta Materialia 52 (8), 709 - 712.

Perez, M., Dumont, M., Acevedo-Reyes, D., 2008. Implementation of classical nucleation and growth theories for precipitation. Acta Materialia $56(9), 2119-2132$. 
Piessens, R., de Doncker-Kapenga, E., Überhuber, C. W., Kahaner, D. K., 2012. Quadpack: A subroutine package for automatic integration. Vol. 1. Springer Science \& Business Media

Povoden-Karadeniz, E., Lang, P., Warczok, P., Falahati, A., Jun, W., Kozeschnik, E., 2013. Calphad modeling of metastable phases in the Al-Mg-Si system. Calphad 43, $94-104$

Radaj, D., 2003. Welding residual stresses and distortion. DVS-Verlag, Dusseldorf.

Reppich, B., 1993. Particle Strengthening. Wiley-VCH.

Slania, J., Mikno, Z., Wojcik, M., 2007. Temperature measurement problems in welding processes. Welding International 21 (8), $589-592$.

Staron, P., Vaidya, W., Koçak, M., 2009. Precipitates in laser beam welded aluminium alloy AA6056 butt joints studied by small-angle neutron scattering. Materials Science and Engineering: A 525 (1), 192-199.

Staron, P., Vaidya, W., Koçak, M., Homeyer, J., Hackius, J., 2006. Residual stresses in laser beam welded butt joints of the airframe aluminium alloy aa6056. In: Materials Science Forum. Vol. 524. Trans Tech Publ, pp. 413-418.

Wagner, R., Kampmann, R., Voorhees, P. W., 2001. Homogeneous Second-Phase Precipitation. Wiley, Ch. 5, pp. 309-407.

Yang, Z., Tao, W., Li, L., Chen, Y., Shi, C., 2017. Numerical simulation of heat transfer and fluid flow during doublesided laser beam welding of t-joints for aluminum aircraft fuselage panels. Optics and Laser Technology 91, 120 - 129. 